\title{
AN ALGEBRAIC APPROACH TO THE REALIZATION OF LOSSLESS NEGATIVE IMAGINARY BEHAVIORS*
}

\author{
SHODHAN RAO ${ }^{\dagger}$ AND PAOLO RAPISARDA ${ }^{\ddagger}$
}

\begin{abstract}
We consider a behavior with an equal number of inputs and outputs for which there exists a quadratic differential form which is positive for nonzero trajectories of the behavior and whose derivative is equal to the scalar product of the input vector and the derivative of the output vector. Such systems occur, for example, when considering conservative mechanical systems. We give a method of computation of a state space realization from an observable image representation of such a behavior. We apply the insights derived from this realization procedure to the synthesis of lossless mechanical systems.
\end{abstract}

Key words. behavioral systems theory, quadratic differential forms, conservative mechanical systems, lossless positive real transfer functions, lossless mechanical network synthesis, time-reversible Hamiltonian systems

AMS subject classifications. 93B20, 93B25, 93B40, 93C05, 93C15, 93C35

DOI. $10.1137 / 100811362$

1. Introduction. We deal with the realization of a class of linear lossless systems with external control $u$ and output $y$ in the form

$$
\begin{aligned}
\left(\begin{array}{c}
\frac{d}{d t} q \\
\frac{d}{d t} p
\end{array}\right) & =\left(\begin{array}{cc}
0 & P \\
-Q & 0
\end{array}\right)\left(\begin{array}{c}
q \\
p
\end{array}\right)+\left(\begin{array}{c}
0 \\
B
\end{array}\right) u, \\
y & =B^{\top} q
\end{aligned}
$$

where $P=P^{\top}>0, Q=Q^{\top}>0$ and the signals $u$ and $y$ have the same dimension.

Equations (1) arise naturally when considering conservative mechanical systems, in which case $q$ is the vector of positions and $p$ that of momenta. In that context, the matrices $P$ and $Q$ are related to the total energy of the system, defined as $\frac{1}{2}\left(p^{\top} P p+q^{\top} Q q\right)=: E(p, q)$; this total energy is conserved in the sense that $\frac{d}{d t} E(p, q)=$ $\frac{1}{2}\left(u^{\top}\left(\frac{d y}{d t}\right)+\left(\frac{d y}{d t}\right)^{\top} u\right)$ for all trajectories $(p, q, u, y)$ satisfying (1), where the functional $\frac{1}{2}\left(u^{\top}\left(\frac{d y}{d t}\right)+\left(\frac{d y}{d t}\right)^{\top} u\right)$ appearing on the right-hand side is the mechanical power. In [7] it has been shown that a state space representation of the form (1) exists for a time-reversible Hamiltonian system, whose transfer function $G \in \mathbb{R}^{\mathrm{u} \times \mathrm{u}}(s)$ is such that

$$
G(s)=G(-s)^{\top}=G(-s),
$$

and a model reduction procedure for such systems has been described in [8].

A representation (1) sometimes needs to be computed from higher-order descriptions of conservative mechanical systems, for example, when the transfer function of

* Received by the editors October 12, 2010; accepted for publication (in revised form) March 19, 2012; published electronically June 26, 2012. A paper with a similar title and by the same authors was presented at the MTNS 2010 conference in Hungary. The contents of the present manuscript are more general and significantly different from those of the paper presented at MTNS 2010.

http://www.siam.org/journals/sicon/50-3/81136.html

${ }^{\dagger}$ Corresponding author. Center for Systems Biology, University of Groningen, Groningen 8747 AG, The Netherlands (shodhanr@googlemail.com).

¥Information: Signals, Images, Systems (ISIS) Research Group, School of Electronics and Computer Science (ECS), University of Southampton, SO17 1BI Southampton, UK (pr3@ecs.soton.ac. uk). 
such a system is specified. In this paper we deal with the computation of a state space representation (1) from a given image representation of the external behavior of (1). Our results consequently enlarge the scope of application of the model reduction procedure of [8] to those situations when the system under consideration is a time-reversible Hamiltonian system described in transfer function or image form.

We use the behavioral framework and the calculus of quadratic differential forms (QDFs); the reader is referred to [4] and [9] for a thorough exposition. We first define a class of behaviors known as lossless negative imaginary behaviors. We then show that a realization of a lossless negative imaginary behavior of the form (1) can be obtained from its image representation using algebraic methods. Our realization procedure produces positive definite matrices $P$ and $Q$; a direct consequence of this fact is that the class of lossless negative imaginary behaviors coincides with the class of Hamiltonian systems investigated in [8]. We also show that when considering single-input, single-output (SISO) lossless negative imaginary behaviors, the matrices $P$ and $Q$ obtained by our realization procedure are also diagonal and tridiagonal, respectively. This special structure of $P$ and $Q$ can be exploited to synthesize in a straightforward way a lossless linear mechanical system from a given transfer function using only springs and masses.

The paper is organized as follows. We review important concepts and algebraic tools in section 2. In section 3, we discuss further properties of QDFs and properties of lossless positive real (LPR) matrices that are relevant for the main result of this paper. We illustrate in section 4 the main result, namely an algorithm to compute a realization (1) of a lossless negative imaginary behavior. In section 5 , we show the application to the synthesis of lossless mechanical systems.

Notation. The space of $n$ dimensional real vectors is denoted by $\mathbb{R}^{\mathrm{n}}$ and the space of $\mathrm{m} \times \mathrm{n}$ real matrices by $\mathbb{R}^{\mathrm{m} \times \mathrm{n}}$. The space of $\mathrm{m} \times \mathrm{m}$ symmetric real matrices is denoted by $\mathbb{R}_{s}^{\mathrm{m} \times \mathrm{m}}$. If one of the dimensions is not specified, a bullet $\bullet$ is used, so that for example, $\mathbb{R}^{\bullet \times n}$ denotes the set of real matrices with $\mathrm{n}$ columns and an unspecified (finite) number of rows. In order to enhance readability, when dealing with a vector space $\mathbb{R}^{\bullet}$ whose elements are denoted with $w$ (respectively, $y, u, x$ ), the notation $\mathbb{R}^{w}$ (respectively $\mathbb{R}^{\mathrm{y}}, \mathbb{R}^{\mathrm{u}}, \mathbb{R}^{\mathrm{x}}$ ) is used, and when dealing with a vector space $\mathbb{R}^{\bullet}$ whose elements are denoted with $\ell$, the notation $\mathbb{R}^{l}$ is used; similar considerations hold for matrices representing linear operators on such spaces. Given two matrices $A$ and $B$ with the same number of columns, we denote with $\operatorname{col}(A, B)$ the matrix obtained by stacking $A$ over $B$; given matrices $R_{1}$ and $R_{2}$ with the same number of rows, we denote with $\operatorname{row}\left(R_{1}, R_{2}\right)$ the matrix obtained by positing the matrix $R_{2}$ to the right of $R_{1}$.

The ring of polynomials with real coefficients in the indeterminate $\xi$ is denoted by $\mathbb{R}[\xi]$; the ring of polynomials with real coefficients in the indeterminates $\zeta$ and $\eta$ is denoted by $\mathbb{R}[\zeta, \eta]$. The set of $\mathrm{n} \times \mathrm{m}$ polynomial matrices in $\xi$ is denoted by $\mathbb{R}^{\mathrm{n} \times \mathrm{m}}[\xi]$ and that consisting of all $\mathrm{n} \times \mathrm{m}$ polynomial matrices in $\zeta$ and $\eta$ by $\mathbb{R}^{\mathrm{n} \times \mathrm{m}}[\zeta, \eta]$. The space of real rational functions in the indeterminate $\xi$ is denoted by $\mathbb{R}(\xi)$ and the space of all matrices of size $\mathrm{w} \times l$, whose entries are real rational functions of the indeterminate $\xi$ is denoted by $\mathbb{R}^{\mathrm{w} \times l}(\xi)$.

The set of infinitely differentiable functions from $\mathbb{R}$ to $\mathbb{R}^{\mathrm{w}}$ is denoted by $\mathfrak{C}^{\infty}\left(\mathbb{R}, \mathbb{R}^{\mathrm{w}}\right)$. The class of behaviors with infinitely differentiable manifest variable $w$ is denoted by $\mathcal{L}^{\text {w. }}$ Given $a_{1}, \ldots, a_{n} \in \mathbb{R}, \operatorname{diag}\left(a_{1}, \ldots, a_{n}\right)$ denotes the diagonal matrix with diagonal entries $a_{1}, \ldots, a_{n}$; this notation is extended to the block-diagonal case when $a_{1}, \ldots, a_{n}$ are real square matrices. $I_{N}$ stands for the identity matrix of size $N .0_{\mathrm{w} \times l}$ denotes a matrix of size $\mathrm{w} \times l$ consisting of zeroes, and $0_{N}$ denotes a square matrix of size $N$ 
consisting of zeroes. $\jmath$ denotes the imaginary square root of -1 . $\Re \mathrm{e}(a)$ and $\Im \mathrm{m}(a)$ denote the real and imaginary parts of a complex number $a . \bar{a}$ denotes the conjugate of a complex number $a$. $A^{*}$ denotes the conjugate transpose of a square complex matrix $A$. The set of positive real numbers is denoted with $\mathbb{R}_{+}$. The set of positive integers is denoted with $\mathbb{Z}_{+}$. The row rank of a matrix $H \in \mathbb{R}^{m \times p}[\xi]$ is denoted by $\operatorname{rank}(H) .\left.M\right|_{A}$ denotes the image of a linear map $M$ with domain restricted to the set $A$.

\section{Background.}

2.1. Linear differential behaviors. A linear differential behavior $\mathfrak{B}$ is a linear subspace of $\mathfrak{C}^{\infty}\left(\mathbb{R}, \mathbb{R}^{\mathrm{w}}\right)$ consisting of all solutions $w$ of a system of linear constantcoefficient differential equations. Such a set is represented as

$$
R\left(\frac{d}{d t}\right) w=0
$$

where $R \in \mathbb{R}^{\bullet \times w}[\xi],(2)$ is called a kernel representation of the behavior $\mathfrak{B}:=\{w \in$ $\mathfrak{C}^{\infty}\left(\mathbb{R}, \mathbb{R}^{\mathrm{w}}\right) \mid w$ satisfies $\left.(2)\right\}$, and $w$ is called the manifest or external variable of $\mathfrak{B}$.

When modeling physical systems from first principles, we often introduce a number of latent (or auxiliary) variables $\ell$ besides the manifest ones; thus latent variable representations

$$
R\left(\frac{d}{d t}\right) w=M\left(\frac{d}{d t}\right) \ell
$$

are obtained. Equation (3) describes the full behavior $\mathfrak{B}_{\mathfrak{f}}:=\left\{(w, \ell) \in \mathfrak{C}^{\infty}\left(\mathbb{R}, \mathbb{R}^{\mathrm{w}+l}\right) \mid\right.$ (3) holds $\}$, and we call the projection of $\mathfrak{B}_{\mathfrak{f}}$ on the $w$ variable, i.e., $\mathfrak{B}:=\{w \in$ $\mathfrak{C}^{\infty}\left(\mathbb{R}, \mathbb{R}^{\mathrm{w}}\right) \mid \exists \ell \in \mathfrak{C}^{\infty}\left(\mathbb{R}, \mathbb{R}^{l}\right)$ such that (3) holds $\}$, the manifest behavior associated with (3).

When $R$ in (3) is the w-dimensional identity matrix $I_{\mathrm{w}}$, we call

$$
w=M\left(\frac{d}{d t}\right) \ell
$$

an image representation of $\mathfrak{B}$. A behavior can be represented by (4) if and only if it is controllable in the behavioral sense (see [4, Chapter 5]). The latent variable $\ell$ in (4) is called observable from $w$ if $[w=0] \Longrightarrow[\ell=0]$. It can be shown that this is the case if and only if the matrix $M(\lambda)$ has full column rank for all $\lambda \in \mathbb{C}$, in which case we speak of an observable image representation. If $\mathfrak{B}$ is controllable, then it always admits an observable image representation (4). Given an image representation, an i/o partition (see [4] for the definition of input and output in the behavioral context) corresponds to a partition of $M$ as $M=\operatorname{col}(U, Y)$ with $U \in \mathbb{R}^{m \times m}[\xi]$ nonsingular. In such a case the transfer function from $u$ to $y$ is the matrix of (not necessarily proper) rational functions $G=Y U^{-1}$. Note that for a controllable system, there always exists an image representation with the number of latent variables equal to the number of inputs of the system.

A behavior $\mathfrak{B}$ is called autonomous if for all $w_{1}, w_{2} \in \mathfrak{B},\left[w_{1}(t)=w_{2}(t)\right.$ for all $t \leq 0] \Longrightarrow\left[w_{1}=w_{2}\right]$. If $\mathfrak{B} \in \mathcal{L}^{\mathrm{w}}$ is autonomous, then it can be proved that there exists $R \in \mathbb{R}^{\mathrm{w} \times \mathrm{w}}[\xi]$ with $\operatorname{det}(R) \neq 0$ such that $\mathfrak{B}=\operatorname{ker}\left(R\left(\frac{d}{d t}\right)\right)$. The roots of the determinant of such an $R$ are called the characteristic frequencies of $\mathfrak{B}$. It can be shown that the characteristic frequencies are independent of the kernel representation $R$ of $\mathfrak{B}$ and consequently are a property of the behavior. 
In this paper we also use the concept of state and of state representation (see [5] for a thorough discussion). A latent variable $\ell$ is a state variable for $\mathfrak{B}$ if and only if $\mathfrak{B}$ admits a representation (3) of first order in $\ell$ and zeroth order in $w: E \frac{d \ell}{d t}+$ $F \ell+G w=0$. Such a representation is called a state representation of $\mathfrak{B}$; in this case we denote the latent variable with $x$. The minimal number of state variables in any state representation of $\mathfrak{B}$ is called the McMillan degree of $\mathfrak{B}$ and is denoted with $\mathrm{n}(\mathfrak{B})$. By combining the notion of state with that of inputs and outputs we arrive at the input/state/output $(i / s / o)$ representation $\frac{d}{d t} x=A x+B u, y=C x+D u$, $w=\operatorname{col}(u, y)$.

For a behavior $\mathfrak{B}$ described in image form, state variables can be computed from the latent variable $\ell$ applying to it a polynomial differential operator called a state map (see [5, section 8]) as in $x=X\left(\frac{d}{d t}\right) \ell$, with $X \in \mathbb{R}^{\mathrm{x} \times l}[\xi]$. We call a state map minimal if it induces a minimal state variable. The problem of computing a state map from an image representation (4) has been dealt with in [5]; in this paper we propose an alternative solution based on two-variable polynomial matrix algebra and energy ideas.

2.2. QDFs. We briefly review the concepts of [9] necessary for the results presented in this paper. A quadratic functional of an infinitely differentiable trajectory $w$ and its derivatives can be written as

$$
Q_{\Phi}(w)=\sum_{h, k=0}^{N}\left(\frac{d^{h} w}{d t^{h}}\right)^{\top} \Phi_{h, k}\left(\frac{d^{k} w}{d t^{k}}\right),
$$

where $\Phi_{h, k} \in \mathbb{R}^{\mathrm{w} \times \mathrm{w}}$ and $N$ is a nonnegative integer. Such a functional is called a QDF. With the QDF (5), we associate the two-variable polynomial matrix

$$
\Phi(\zeta, \eta)=\sum_{h, k=0}^{N} \Phi_{h, k} \zeta^{h} \eta^{k} .
$$

Without loss of generality, when considering QDFs we may assume that $\Phi \in \mathbb{R}^{\mathrm{w} \times \mathrm{w}}[\zeta, \eta]$ is symmetric, i.e., $\Phi(\zeta, \eta)=\Phi(\eta, \zeta)^{\top}$; we denote the set of all such matrices with $\mathbb{R}_{s}^{\mathrm{W} \times \mathrm{w}}[\zeta, \eta]$.

A QDF $Q_{\Psi}$ is called the derivative of a $\operatorname{QDF} Q_{\Phi}$ if for all $w \in \mathfrak{C}^{\infty}\left(\mathbb{R}, \mathbb{R}^{\mathrm{w}}\right)$ it holds that $\frac{d}{d t}\left(Q_{\Phi}(w)\right)=Q_{\Psi}(w)$; for the corresponding two-variable polynomial matrices, there holds $(\zeta+\eta) \Phi(\zeta, \eta)=\Psi(\zeta, \eta)$ (see $[9]$ ).

In this paper the notions of nonnegativity and positivity of QDFs play an important role.

Definition 1. Let $\Phi \in \mathbb{R}_{s}^{\mathrm{w} \times \mathrm{w}}[\zeta, \eta]$. $Q_{\Phi}$ is said to be nonnegative, denoted by $Q_{\Phi} \geq 0$ if $Q_{\Phi}(w) \geq 0$ for all $w \in \mathfrak{C}^{\infty}\left(\mathbb{R}, \mathbb{R}^{w}\right)$, and positive, denoted by $Q_{\Phi}>0$, if $Q_{\Phi} \geq 0$ and $\left[Q_{\Phi}(w)=0\right] \Longrightarrow[w=0]$.

When considering the interplay of dynamics and functionals, we consider QDFs restricted to the trajectories of a given behavior $\mathfrak{B}$. The definition of nonnegative or positive QDF along a behavior is as follows.

Definition 2. Let $\Phi \in \mathbb{R}_{s}^{\mathrm{W} \times \mathrm{w}}[\zeta, \eta]$. $Q_{\Phi}$ is said to be nonnegative along $\mathfrak{B}$, denoted by $Q_{\Phi} \stackrel{\mathfrak{B}}{\stackrel{\mathfrak{B}}{\geq}} 0$, if $Q_{\Phi}(w) \geq 0$ for all $w \in \mathfrak{B}$, and positive along $\mathfrak{B}$, denoted by $Q_{\Phi} \stackrel{\mathfrak{B}}{>} 0$, if $Q_{\Phi} \geq 0$ and $\left[Q_{\Phi}(w)=0\right.$ and $\left.w \in \mathfrak{B}\right] \Longrightarrow[w=0]$.

These properties translate to properties of the two-variable polynomial matrices inducing the QDFs as follows. 
Proposition 3. Let $\Phi \in \mathbb{R}_{s}^{\mathrm{w} \times \mathrm{w}}[\zeta, \eta]$ and let $\mathfrak{B}=\operatorname{ker}\left(R\left(\frac{d}{d t}\right)\right)$. Then

1. $Q_{\Phi} \stackrel{\mathfrak{B}}{\geq} 0$ if and only if there exists $F \in \mathbb{R}^{\bullet \times \mathrm{w}}[\zeta, \eta]$ and $D \in \mathbb{R}^{\bullet \times \mathrm{w}}[\xi]$ such that

$$
\Phi(\zeta, \eta)=D(\zeta)^{\top} D(\eta)+F(\eta, \zeta)^{\top} R(\eta)+R(\zeta)^{\top} F(\zeta, \eta)
$$

2. $Q_{\Phi} \stackrel{\mathfrak{B}}{>} 0$ if and only if $Q_{\Phi} \stackrel{\mathfrak{B}}{\geq} 0$ and $\operatorname{col}(D(\lambda), R(\lambda))$ has full column rank for all $\lambda \in \mathbb{C}$.

Proof. See [9, Proposition 3.5].

2.3. LPR and lossless negative imaginary transfer functions. In this section, we define LPR and lossless negative imaginary transfer functions as in [1] and [10], respectively. These concepts are needed to understand the results presented in this paper. We begin with the definition of a positive real transfer function.

Definition 4. A rational matrix $B \in \mathbb{R}^{\mathrm{u} \times \mathrm{u}}(\xi)$ is called positive real if the following conditions hold:

1. All elements of $B$ are analytic in the open right half plane.

2. $B(\lambda)^{*}+B(\lambda) \geq 0$ for $\operatorname{Re}(\lambda)>0$.

Given below is the definition of a LPR transfer function.

Definition 5. A rational matrix $B \in \mathbb{R}^{\mathrm{u} \times \mathrm{u}}(\xi)$ is called $L P R$ if the following conditions hold:

1. $B$ is positive real.

2. $B(j \omega)^{*}+B(j \omega)=0$ for all $\omega \in \mathbb{R}$ with $j \omega$ not a pole of any element of $B$.

Some properties of positive real functions are the following:

1. The sum of two positive real functions is also positive real.

2. If $B \in \mathbb{R}^{\mathrm{u} \times \mathrm{u}}(\xi)$ is positive real, then $B^{-1}$ is also positive real if it exists. (For a proof, see [3, Theorem $5-8$, p. 126]). Moreover if $B$ is LPR, then so is $B^{-1}$ if it exists.

Lossless negative imaginary transfer functions were first defined in [10].

Definition 6. A rational matrix $G \in \mathbb{R}^{\mathrm{u} \times \mathrm{u}}(\xi)$ is called lossless negative imaginary if $H$ defined by $H(\xi):=\xi G(\xi)$ is $L P R$.

3. Further properties of QDFs and LPR matrices. In this section, we discuss a property of a QDF that plays an important role in the main result of our paper. We also discuss some properties of LPR matrices that are required to prove our main result.

TheOREm 7. Let $\Psi \in \mathbb{R}_{s}^{\mathrm{w} \times \mathrm{w}}[\zeta, \eta]$ be such that $Q_{\Psi} \geq 0$. Assume that $\Phi(\zeta, \eta):=$ $(\zeta+\eta) \Psi(\zeta, \eta)$ can be factored as $\Phi(\zeta, \eta)=Y(\zeta)^{\top} \mathcal{I}(\eta)+\mathcal{I}(\zeta)^{\top} Y(\eta)$ with $\mathcal{I}$ square and nonsingular and all elements of $Z:=Y \mathcal{I}^{-1}$ analytic in the open right half plane. Then the rational function $Z$ is $L P R$.

Proof. We make use of the concept half-line nonnegativity of QDFs described in [9, pp. 1725-1726]. It can be proved (see [9, Theorem 6.3]) that a QDF $Q_{\Phi}$ is half-line nonnegative if and only if there exists $Q_{\Psi} \geq 0$ such that $\frac{d}{d t} Q_{\Psi} \leq Q_{\Phi}$. Consequently, the QDF $Q_{\Phi}$ in the statement of Theorem 7 is half-line nonnegative. From [9, Proposition 6.2], it follows then also that $\Phi(\bar{\lambda}, \lambda) \geq 0$ for all $\lambda \in \mathbb{C}$ with $\Re \mathrm{e}(\lambda)>0$.

This implies that $Z(\lambda)^{*}+Z(\lambda) \geq 0$ for all $\lambda \in \mathbb{C}$ with $\Re \mathrm{e}(\lambda)>0$ such that $\lambda$ is not a pole of $Z$. Since $\Phi(-\jmath \omega, \jmath \omega)=0$ for all $\omega \in \mathbb{R}$, we have $Z(-\jmath \omega)^{\top}+Z(\jmath \omega)=0$ for all $\omega \in \mathbb{R}$ such that $j \omega$ is not a pole of $Z$. From Definition 5 , it follows that $Z$ is LPR.

The following theorem will be instrumental in proving the main result of this paper. It elaborates on the Foster partial fraction expansion of an LPR transfer 
function. It will be used later to obtain a state space realization and a corresponding state map of a lossless negative imaginary behavior starting from an observable image representation of the behavior.

Theorem 8. Let $N, D \in \mathbb{R}^{l \times l}[\xi]$ be nonsingular. Define $Z:=D N^{-1}$. Assume that $Z^{-1}$ is strictly proper, $Z(\xi)=Z(-\xi)$, and $Z^{\prime}(\xi):=\frac{Z(\xi)}{\xi}$ is LPR. Also assume that $D(0)$ has full rank.

1. $Z$ can be written as

$$
Z(\xi)=A \xi^{2}+B-\sum_{i=1}^{p} \frac{C_{i}}{\xi^{2}+\omega_{i}^{2}},
$$

where $A, B \in \mathbb{R}_{s}^{l \times l}$ are positive definite, $C_{i} \in \mathbb{R}_{s}^{l \times l}$ are positive semidefinite for $i=1, \ldots, p$, and $\omega_{i} \in \mathbb{R}_{+}$are such that $\omega_{i} \neq \omega_{j}, i \neq j$, for $i=1, \ldots, p$. Moreover, $Z(0)$ is positive definite.

2. $N(0)$ has full rank.

3. $N_{1}(\xi):=\left(A \xi^{2}+B\right) N(\xi)-D(\xi)$ is such that $Z_{1}:=N_{1} N^{-1}$ is strictly proper, $Z_{1}(\xi)=Z_{1}(-\xi)$, and $Z_{1}^{\prime}(\xi):=\xi Z_{1}(\xi)$ is $L P R$.

Proof. (1) In [3, pp. 122-123], it is shown that $Z^{\prime} \in \mathbb{R}^{l \times l}(\xi)$ is LPR if and only if it has a Foster partial fraction expansion given by

$$
Z^{\prime}(\xi)=J+\xi A+\frac{K_{0}}{\xi}+\sum_{i=1}^{p}\left(\frac{\xi D_{i}+E_{i}}{\xi^{2}+\omega_{i}^{2}}\right)
$$

where $A, K_{0}, D_{i} \in \mathbb{R}_{s}^{l \times l}$ are nonnegative definite, $J, E_{i} \in \mathbb{R}^{l \times l}$ are skew symmetric, and $\omega_{i} \in \mathbb{R}_{+}$are distinct for $i=1, \ldots, p$. Consequently

$$
Z(\xi)=\xi Z^{\prime}(\xi)=J \xi+A \xi^{2}+K_{0}+\sum_{i=1}^{p}\left(\frac{\xi^{2} D_{i}+E_{i} \xi}{\xi^{2}+\omega_{i}^{2}}\right) .
$$

Since $Z(\xi)=Z(-\xi)$, we get $J=0$ and $E_{i}=0$ for $i=1, \ldots, p$. Thus

$$
Z(\xi)=A \xi^{2}+\left(K_{0}+\sum_{i=1}^{p} D_{i}\right)-\sum_{i=1}^{p} \frac{D_{i} \omega_{i}^{2}}{\xi^{2}+\omega_{i}^{2}} .
$$

Now define $B:=K_{0}+\sum_{i=1}^{p} D_{i}$ and $C_{i}:=D_{i} \omega_{i}^{2}$ for $i=1, \ldots, p$ to get (6).

We now prove that $A$ is positive definite. Observe that

$$
D(\xi)=A \xi^{2} N(\xi)+\left[B-\sum_{i=1}^{p} \frac{C_{i}}{\xi^{2}+\omega_{i}^{2}}\right] N(\xi) .
$$

Since $D^{-1}$ exists, it follows that $D$ has full column rank. Consequently, there exists a unimodular $V \in \mathbb{R}^{l \times l}[\xi]$ such that $D^{\prime}:=D V$ is column reduced (see $[2$, p. 386]). Define $N^{\prime}:=N V$. Then

$$
D^{\prime}(\xi)=A \xi^{2} N^{\prime}(\xi)+\left[B-\sum_{i=1}^{p} \frac{C_{i}}{\xi^{2}+\omega_{i}^{2}}\right] N^{\prime}(\xi)
$$

Since $Z^{-1}=N^{\prime} D^{\prime-1}$ is strictly proper, it follows that every column of $D^{\prime}$ has degree higher than that of the corresponding column of $N^{\prime}$ (see [2, Lemma 6.3-10, p. 383]).

Copyright (c) by SIAM. Unauthorized reproduction of this article is prohibited. 
This implies that the highest degree terms of every column of $D^{\prime}$ are the same as those of the first term on the right-hand side of (8). Let $n_{i}$ denote the degree of the ith column of $D^{\prime}$. Then we can write

$$
D^{\prime}(\xi)=D_{\mathrm{hc}}^{\prime} \operatorname{diag}\left(\xi^{n_{1}}, \xi^{n_{2}}, \ldots, \xi^{n_{l}}\right)+B_{1}(\xi)
$$

where $D_{\mathrm{hc}}^{\prime}$ is the coefficient matrix of the polynomial matrix formed by the highest degree terms of $D^{\prime}$ and $B_{1} \in \mathbb{R}^{l \times l}[\xi]$ consists of the remaining lower degree terms of $D^{\prime}$. From the previous discussion, it follows that $D_{\mathrm{hc}}^{\prime}=A A_{1}$ for some $A_{1} \in \mathbb{R}^{l \times l}$. Since $D^{\prime}$ is column reduced, it follows that $D_{\mathrm{hc}}^{\prime}=A A_{1}$ has full rank. Consequently $A$ is positive definite.

We now prove that $Z(0)$ and $B$ are positive definite. From (7), it follows that $K_{0}=Z(0)$. This implies that $D(0)=K_{0} N(0)$. Now assume by contradiction that $\operatorname{det}\left(K_{0}\right)=0$. This implies that there exists a $v \in \mathbb{R}^{1 \times l}$ such that $v K_{0}=0$; but then also $v D(0)=0$ which is a contradiction $(D(0)$ is assumed to have full rank). Consequently $K_{0}=Z(0)$ is positive definite and so is $B$.

(2) From (7), it follows that $D(0)=K_{0} N(0)$. Since $D(0)$ and $K_{0}$ have full rank, it follows that $N(0)$ also has full rank.

(3) Observe that $Z_{1}(\xi)=\sum_{i=1}^{p} \frac{C_{i}}{\xi^{2}+\omega_{i}^{2}}$; this is a partial fraction expansion for $Z_{1}$ with $C_{i} \geq 0$ for $i=1, \ldots, p$. From the results in [3, pp. 122-123], it follows that $Z_{1}^{\prime}$ is LPR. The other properties of $Z_{1}$ mentioned in the statement of the theorem can be verified easily.

Remark 9. Note that in the SISO case, $Z$ being strictly proper implies that $Z(\xi)=Z(-\xi)$, as $J$ and $E_{i}$ in (7) are equal to 0 owing to their being scalar and skew symmetric. Consequently for the SISO case the statement $Z(\xi)=Z(-\xi)$ in Theorem 8 is redundant.

Remark 10. In Theorem $8, Z$ satisfies $Z(\xi)=Z(-\xi)=Z(-\xi)^{\top}$. A system with such transfer function is a time-reversible Hamiltonian system. From the proof of Theorem 8, it follows that the special structure of the Foster expansion (6) is mainly due to this implicit assumption of time-reversibility.

We conclude this section with the following important result.

Proposition 11. Let $Y, \mathcal{I} \in \mathbb{R}^{l \times l}[\xi]$ be such that $\mathcal{I}$ is invertible and $Z(\xi):=$ $\xi Y(\xi) \mathcal{I}(\xi)^{-1}$ is LPR. Assume that for some $\lambda \in \mathbb{R}_{+}, Y(\lambda)$ has rank $l_{1}<l$. Assume that $T \in \mathbb{R}^{l \times l}$ is invertible and such that $T Y(\lambda)=\operatorname{col}\left(Y_{1}(\lambda), 0_{\left(l-l_{1}\right) \times l}\right)$ with $Y_{1} \in$ $\mathbb{R}^{l_{1} \times l}[\xi]$. Partition $T Y(\xi)$ as $T Y(\xi)=\operatorname{col}\left(Y_{1}(\xi), Y_{2}(\xi)\right)$, where $Y_{2} \in \mathbb{R}^{\left(l-l_{1}\right) \times l}[\xi]$. Then $Y_{2}(\xi)=0_{\left(l-l_{1}\right) \times l}$ identically.

Proof. We make use of the following result from [3].

Lemma 12. Let $A \in \mathbb{R}^{l \times l}(\xi)$ be positive real. Consider a partition of $A$ given by

$$
A(\xi)=\left[\begin{array}{ll}
A_{11}(\xi) & A_{12}(\xi) \\
A_{21}(\xi) & A_{22}(\xi)
\end{array}\right]
$$

where $A_{11} \in \mathbb{R}^{l_{1} \times l_{1}}(\xi), \quad A_{12} \in \mathbb{R}^{l_{1} \times\left(l-l_{1}\right)}(\xi), \quad A_{21} \in \mathbb{R}^{\left(l-l_{1}\right) \times l_{1}}(\xi)$, and $A_{22} \in \mathbb{R}^{\left(l-l_{1}\right) \times\left(l-l_{1}\right)}(\xi)$. Assume that $\exists \lambda \in \mathbb{R}_{+}$such that $A_{21}(\lambda)=0_{\left(l-l_{1}\right) \times l_{1}}$ and $A_{22}(\lambda)=0_{\left(l-l_{1}\right) \times\left(l-l_{1}\right)}$. Then $A_{21}(\xi)=0_{\left(l-l_{1}\right) \times l_{1}}, A_{22}(\xi)=0_{\left(l-l_{1}\right) \times\left(l-l_{1}\right)}$, and $A_{12}(\xi)=0_{l_{1} \times\left(l-l_{1}\right)}$.

Proof. Can be deduced from the proof of [3, Theorem 5-11, pp. 128-129].

We now resume the proof of the proposition. Observe that $T Z(\xi) T^{\top}$ is positive real and that $T Z(\lambda) T^{\top}$ has the last $\left(l-l_{1}\right)$ rows zero. From Lemma 12, it follows that $T Z(\xi) T^{\top}$ has its last $\left(l-l_{1}\right)$ rows identically zero, which in turn implies that $Y_{2}(\xi)=0_{\left(l-l_{1}\right) \times l}$. This concludes the proof. 
4. Main result. We begin with the definition of lossless negative imaginary behavior. Hereafter $J$ is defined as

$$
J(\zeta, \eta):=\frac{1}{2}\left[\begin{array}{cc}
0_{l} & \zeta I_{l} \\
\eta I_{l} & 0_{l}
\end{array}\right]
$$

Definition 13 (lossless negative imaginary behavior). $\mathfrak{B} \in \mathcal{L}^{2 l}$ is lossless negative imaginary if there exists a $Q D F Q_{E} \stackrel{\mathfrak{B}}{>} 0$ such that for every $w \in \mathfrak{B}$, $Q_{J}(w)=\frac{d}{d t} Q_{E}(w) . Q_{E}(w)$ is called the principal energy associated with a trajectory $w \in \mathfrak{B}$.

$Q_{J}$ is to be interpreted as the power entering the system; when considering conservative mechanical systems with external variable $\operatorname{col}(y, u)$ with $y$ and $u$ having the same dimension $l$, the power is the scalar product of the input vector (force or torque) and the derivative of the output vector (linear or angular displacement).

imaginary (see Appendix for a definition). This is the reason for the the nomenclature "lossless negative imaginary" in Definition 13.

The following result shows how to obtain an input-output partition of a lossless negative imaginary behavior and shows that under suitable conditions the corresponding transfer function is lossless negative imaginary, thus justifying the nomenclature "lossless negative imaginary" in Definition 13.

Lemma 14. Consider a controllable lossless negative imaginary $\mathfrak{B} \in \mathcal{L}^{2 l}$ with an observable image representation

$$
w=\left[\begin{array}{l}
w_{1} \\
w_{2}
\end{array}\right]=\left[\begin{array}{l}
N\left(\frac{d}{d t}\right) \\
D\left(\frac{d}{d t}\right)
\end{array}\right] \ell,
$$

where $\operatorname{dim}\left(w_{1}\right)=\operatorname{dim}\left(w_{2}\right)=l$ and $N, D \in \mathbb{R}^{l \times l}[\xi]$. Then $N$ and $D$ are both invertible and $\operatorname{col}\left(w_{1}, w_{2}\right)$ is an output-input partition of $\mathfrak{B}$. Further if $Z:=N D^{-1}$ is analytic in the open right half plane, then it is lossless negative imaginary.

Proof. First, we prove that $D$ and $N$ are both invertible. Define $M:=\operatorname{col}(N, D)$,

$$
\Phi(\zeta, \eta):=M(\zeta)^{\top} J(\zeta, \eta) M(\eta)=\frac{\zeta N(\zeta)^{\top} D(\eta)+D(\zeta)^{\top} N(\eta) \eta}{2} .
$$

Let $E \in \mathbb{R}^{2 l \times 2 l}[\zeta, \eta]$ be such that $Q_{E}(w)$ is the principal energy associated with $w \in \mathfrak{B}$. Observe that $Q_{J}(w)=Q_{\Phi}(\ell)=\frac{d}{d t} Q_{E}(w)=\frac{d}{d t} Q_{E^{\prime}}(\ell)$ for every $(w, \ell)$ related as $w=M\left(\frac{d}{d t}\right) \ell$, with

$$
E^{\prime}(\zeta, \eta)=M(\zeta)^{\top} E(\zeta, \eta) M(\eta)=\frac{\zeta N(\zeta)^{\top} D(\eta)+D(\zeta)^{\top} N(\eta) \eta}{2(\zeta+\eta)}
$$

Note that since $Q_{E} \stackrel{\mathfrak{B}}{>} 0$ and $\ell$ can be chosen arbitrarily, $Q_{E^{\prime}}>0$. Now assume by contradiction that $D$ is not invertible. Then there exists a nonzero $v \in \mathbb{R}^{l}[\xi]$ for which $D(\xi) v(\xi)=0$. After multiplication of $E^{\prime}(\zeta, \eta)$ on the left by $v(\zeta)^{\top}$ and on the right by $v(\eta)$, we obtain zero. Since $Q_{E^{\prime}}>0$, from Proposition 3 it follows that there exists $D^{\prime} \in \mathbb{R}^{\bullet \times l}[\xi]$ such that $E^{\prime}(\zeta, \eta)=D^{\prime}(\zeta)^{\top} D^{\prime}(\eta)$ with $D^{\prime}(\lambda)$ having full column rank for all $\lambda \in \mathbb{C}$. This implies that $v(\zeta)^{\top} D^{\prime}(\zeta)^{\top} D^{\prime}(\eta) v(\eta)=0$, which in turn implies that $D^{\prime}(\xi)$ does not have full column rank, a contradiction. This proves that $D$ is invertible. We can prove that $N$ is invertible by an analogous argument.

Since $D$ is invertible, from the discussion of section 2.1 it follows that $w=$ $\operatorname{col}\left(w_{1}, w_{2}\right)$ is an output-input partition of $\mathfrak{B}$. Define $F(\xi):=\xi Z(\xi)$. It is easy to see 
that all elements of $F$ are analytic in the open right half plane. Since $Q_{E^{\prime}}>0$ and $\frac{d}{d t} Q_{E^{\prime}}=Q_{\Phi}$, from Theorem 7 it follows that $F$ is LPR. Consequently, by definition, $Z$ is lossless negative imaginary.

We now show that if $\mathfrak{B}$ is a lossless negative imaginary behavior with an observable image representation $w=L\left(\frac{d}{d t}\right) \ell$ satisfying some additional properties and $Q_{E^{\prime}}(\ell)$ is the principal energy associated with a trajectory $w=L\left(\frac{d}{d t}\right) \ell \in \mathfrak{B}$, then $E^{\prime}$ can be written as

$$
E^{\prime}(\zeta, \eta)=\frac{1}{2} X^{\prime}(\zeta)^{\top}\left[\begin{array}{cc}
K & 0 \\
0 & M^{-1}
\end{array}\right] X^{\prime}(\eta)
$$

where $X^{\prime}(\xi)$ is a state map for $\mathfrak{B}$ with a special structure, $K=K^{\top}>0$, and $M=M^{\top}>0$ is block-diagonal. From such $X^{\prime}(\xi)$, a realization (1) can be readily obtained.

THEOREM 15. Let $w=\left(\begin{array}{l}N_{0}\left(\frac{d}{d t}\right) \\ D_{0}\left(\frac{d}{d t}\right)\end{array}\right) \ell$ with $N_{0}, D_{0} \in \mathbb{R}^{l \times l}[\xi]$ be an observable image representation of a lossless negative imaginary behavior $\mathfrak{B}$. Define $L:=\operatorname{col}\left(N_{0}, D_{0}\right)$, $B:=\operatorname{col}\left(I_{l}, 0_{(N-l) \times l}\right), y:=N_{0}\left(\frac{d}{d t}\right) \ell, u:=D_{0}\left(\frac{d}{d t}\right) \ell$, and

$$
\mathfrak{B}_{y}:=\left\{y \in \mathfrak{C}^{\infty}\left(\mathbb{R}, \mathbb{R}^{l}\right) \mid \operatorname{col}\left(y, 0_{l \times 1}\right) \in \mathfrak{B}\right\} .
$$

Assume that

1. $\mathfrak{B}_{y}$ does not have zero among its characteristic frequencies;

2. $\left[w \in \mathfrak{B}_{y}\right] \Longrightarrow[w$ is bounded on $[0, \infty)]$;

3. $Z:=N_{0} D_{0}^{-1}$ is strictly proper;

4. $\mathfrak{B}$ is time-reversible, i.e., if $w \in \mathfrak{B}$, then the time-reversed trajectory $w^{\prime}$ defined by $w^{\prime}(t):=w(-t)$ is also a trajectory of $\mathfrak{B}$.

Then there exists $N \in \mathbb{Z}_{+}$, a positive definite block-tridiagonal matrix $K \in \mathbb{R}_{s}^{N \times N}$, a positive definite block-diagonal matrix $M \in \mathbb{R}_{s}^{N \times N}$, and $X \in \mathbb{R}^{N \times l}[\xi]$ such that the following hold:

(i) $E^{\prime}(\zeta, \eta)=\frac{1}{2}\left(X(\zeta)^{\top} K X(\eta)+\zeta \eta X(\zeta)^{\top} M X(\eta)\right)$ is such that $Q_{E^{\prime}}(\ell)$ is the principal energy associated with a trajectory $w=L\left(\frac{d}{d t}\right) \ell \in \mathfrak{B}$.

(ii) The following is a state space representation of $\mathfrak{B}$ :

$$
\begin{aligned}
\frac{d}{d t}\left[\begin{array}{l}
q \\
p
\end{array}\right] & =\left[\begin{array}{cc}
0_{N} & M^{-1} \\
-K & 0_{N}
\end{array}\right]\left[\begin{array}{l}
q \\
p
\end{array}\right]+\left[\begin{array}{c}
0_{N \times l} \\
B
\end{array}\right] u, \\
y & =B^{\top} q,
\end{aligned}
$$

where $q:=X\left(\frac{d}{d t}\right) \ell$ and $p:=\frac{d}{d t}\left(M X\left(\frac{d}{d t}\right) \ell\right) \cdot \operatorname{col}(X(\xi), \xi M X(\xi))$ is a state map for $\mathfrak{B}$.

Remark 16. Before giving the proof of the theorem, we discuss assumption 1. Observe that $\mathfrak{B}_{y}=\left.N_{0}\left(\frac{d}{d t}\right)\right|_{\operatorname{ker}\left(D_{0}\left(\frac{d}{d t}\right)\right)}$. Assumption 1 is equivalent to saying that $D_{0}(0)$ has full rank, or equivalently that there does not exist a trajectory of $\mathfrak{B}$ in which zero input corresponds to a nonzero constant output.

Note that we would like to relate lossless negative imaginary behaviors with behaviors of mechanical systems whose governing differential equations are given by (10), where $M=M^{\top}>0, K=K^{\top}>0$ are the mass and stiffness matrices of the system, respectively, $q$ and $p$ denote the vectors of positions and momenta, respectively, and the dimensions of external variables $y$ and $u$ are each equal to $l$. Now consider a trajectory $\operatorname{col}\left(y_{c}, 0_{l \times 1}\right)$, where $y_{c}(t) \in \mathbb{R}^{l}$ is a nonzero constant for all $t \in \mathbb{R}$. Let $q_{c}$ be the corresponding position vector. Since $y_{c}=B^{\top} q_{c}$, it is easy to see that $q_{c} \neq 0$. 
Since $M>0$, it follows that $M q_{c} \neq 0$, which in turn implies that the center of mass of the system is not at the origin. However, under zero input, we would like the center of mass of the system to be fixed at the origin. Consequently in order to accommodate such mechanical systems, we consider lossless negative imaginary behaviors that obey Assumption 1.

Proof. We give a constructive proof of statement (i), i.e., we illustrate a procedure for deriving $X, M$, and $K$ such that $E^{\prime}(\zeta, \eta)=\frac{1}{2}\left(X(\zeta)^{\top} K X(\eta)+\zeta \eta X(\zeta)^{\top} M X(\eta)\right)$.

Since $\mathfrak{B}_{y}=\left.N_{0}\left(\frac{d}{d t}\right)\right|_{\operatorname{ker}\left(D_{0}\left(\frac{d}{d t}\right)\right)}$ is bounded on $[0, \infty)$, it follows that there is no characteristic frequency of $\mathfrak{B}_{y}$ in the open right half plane; this implies that every entry of $Z$ is analytic in the open right half plane. Define

$$
\Phi_{0}(\zeta, \eta):=L(\zeta)^{\top} J(\zeta, \eta) L(\eta)=\frac{\zeta N_{0}(\zeta)^{\top} D_{0}(\eta)+D_{0}(\zeta)^{\top} N_{0}(\eta) \eta}{2} .
$$

Let $E \in \mathbb{R}^{2 l \times 2 l}[\zeta, \eta]$ be such that $Q_{E}(w)$ is the principal energy associated with $w \in \mathfrak{B}$. Observe that $Q_{J}(w)=Q_{\Phi}(\ell)=\frac{d}{d t} Q_{E}(w)=\frac{d}{d t} Q_{E^{\prime}}(\ell)$ for every $(w, \ell)$ related as $w=L\left(\frac{d}{d t}\right) \ell$ with

$$
E^{\prime}(\zeta, \eta)=L(\zeta)^{\top} E(\zeta, \eta) L(\eta)=\frac{\zeta N_{0}(\zeta)^{\top} D_{0}(\eta)+D_{0}(\zeta)^{\top} N_{0}(\eta) \eta}{2(\zeta+\eta)}
$$

Note that since $Q_{E} \stackrel{\mathfrak{B}}{>} 0$ and $\ell$ can be chosen arbitrarily, $Q_{E^{\prime}}>0$. From Theorem 7, it follows that $\xi Z(\xi)$ is LPR. From Lemma 14, it follows that $N_{0}$ and consequently $Z$ are invertible. This implies that $\frac{Z(\xi)^{-1}}{\xi}$ is also LPR. Since $\mathfrak{B}$ is time-reversible, it follows that $Z(\xi)=Z(-\xi)$. Consequently, from Theorem 8 , it follows that we can write

$$
D_{0}(\xi)=\left(A_{0} \xi^{2}+B_{0}\right) N_{0}(\xi)-N_{1}(\xi)
$$

where $A_{0}, B_{0} \in \mathbb{R}_{s}^{l \times l}$ are positive definite, $N_{1} \in \mathbb{R}^{l \times l}[\xi], F_{1}(\xi):=\xi N_{1}(\xi) N_{0}(\xi)^{-1}$ is LPR, $N_{0}(0)$ has full rank, and $Z_{1}:=N_{1} N_{0}^{-1}$ is strictly proper and satisfies $Z_{1}(\xi)=$ $Z_{1}(-\xi)$. Define

$$
E_{1}(\zeta, \eta):=\frac{\zeta N_{1}(\zeta)^{\top} N_{0}(\eta)+N_{0}(\zeta)^{\top} N_{1}(\eta) \eta}{2(\zeta+\eta)}
$$

and observe that

$$
\begin{aligned}
E^{\prime}(\zeta, \eta) & =\frac{\zeta N_{0}(\zeta)^{\top} D_{0}(\eta)+D_{0}(\zeta)^{\top} N_{0}(\eta) \eta}{2(\zeta+\eta)} \\
& =\frac{1}{2}\left(N_{0}(\zeta)^{\top}\left(A_{0} \zeta \eta+B_{0}\right) N_{0}(\eta)-N_{0}(\zeta)^{\top} N_{1}(\eta)-N_{1}(\zeta)^{\top} N_{0}(\eta)\right)+E_{1}(\zeta, \eta) .
\end{aligned}
$$

The remainder of the argument pivots on the extraction from $E_{1}(\zeta, \eta)$ of an image representation of a lower McMillan degree system satisfying the assumptions of the theorem. The decomposition of the principal energy function can then be applied recursively, and from such decompositions the matrices $M, K$, and $X$ can be easily computed.

To begin with, we prove that $\operatorname{col}\left(N_{1}, N_{0}\right)$ is observable. By contradiction if there exists $\lambda \in \mathbb{C}$ and $v \in \mathbb{C}^{l}$ such that $N_{1}(\lambda) v=0$ and $N_{0}(\lambda) v=0$, then from (11) it follows that also $D_{0}(\lambda) v=0$, contradicting observability of $\operatorname{col}\left(N_{0}, D_{0}\right)$.

Copyright $@$ by SIAM. Unauthorized reproduction of this article is prohibited. 
Now consider two cases.

Case 1. $\operatorname{det}\left(N_{1}\right) \neq 0$. This implies that $Z_{1}$ is invertible. It follows that $F_{1}(\xi)^{-1}=$ $\frac{Z_{1}(\xi)^{-1}}{\xi}$ is LPR. Since $N_{0}(0)$ has full rank, $Z_{1}$ is strictly proper and obeys $Z_{1}(\xi)=$ $Z_{1}(-\xi)$. From Theorem 8 it follows that we can compute $N_{2} \in \mathbb{R}^{l \times l}[\xi]$ and positive definite $A_{1}, B_{1} \in \mathbb{R}_{s}^{l \times l}$ such that $F_{2}(\xi):=\xi N_{2}(\xi) N_{1}(\xi)^{-1}$ is LPR, $Z_{2}:=N_{2} N_{1}^{-1}$ is strictly proper and obeys $Z_{2}(\xi)=Z_{2}(-\xi)$. Moreover,

$$
\begin{aligned}
N_{0}(\xi)= & \left(A_{1} \xi^{2}+B_{1}\right) N_{1}(\xi)-N_{2}(\xi) . \\
E_{1}(\zeta, \eta)= & \frac{1}{2}\left(N_{1}(\zeta)^{\top}\left(A_{1} \zeta \eta+B_{1}\right) N_{1}(\eta)-N_{1}(\zeta)^{\top} N_{2}(\eta)-N_{2}(\zeta)^{\top} N_{1}(\eta)\right) \\
& +E_{2}(\zeta, \eta),
\end{aligned}
$$

where

$$
E_{2}(\zeta, \eta):=\frac{\zeta N_{2}(\zeta)^{\top} N_{1}(\eta)+N_{1}(\zeta)^{\top} N_{2}(\eta) \eta}{2(\zeta+\eta)}
$$

From Theorem 8, it follows that $N_{1}(0)$ has full rank. Since $\operatorname{col}\left(N_{1}, N_{0}\right)$ is observable, from (12) it follows that $\operatorname{col}\left(N_{2}, N_{1}\right)$ is also observable.

Case 2. $\operatorname{det}\left(N_{1}\right)=0$. Then $l_{1}:=\operatorname{rank}\left(N_{1}\right)<l$. In the following, the two-variable polynomial matrix $E_{1}$ will be transformed to a matrix with all entries equal to zero except the top left $\left(l_{1} \times l_{1}\right)$ block. This will be effected by pre- and post-multiplication by a suitable unimodular matrix $V$

$$
V(\zeta)^{\top} E_{1}(\zeta, \eta) V(\eta)=\left[\begin{array}{cc}
\tilde{E}_{1}(\zeta, \eta) & 0_{l_{1} \times\left(l-l_{1}\right)} \\
0_{\left(l-l_{1}\right) \times l_{1}} & 0_{\left(l-l_{1}\right) \times\left(l-l_{1}\right)}
\end{array}\right]
$$

Since $N_{1}$ has rank $l_{1}$, there exists a unimodular matrix $U \in \mathbb{R}^{l \times l}[\xi]$ such that $U N_{1}$ has its last $l-l_{1}$ rows filled with zeroes. Define $T:=U(\lambda)$, where $\lambda$ is any positive real number. From Proposition 11, it follows that $R(\xi):=T N_{1}(\xi)=\operatorname{col}\left(Y_{1}(\xi), 0_{\left(l-l_{1}\right) \times l}\right)$. Since $N_{1}$ has rank $l_{1}$, it follows that $Y_{1}$ has full row rank. Now consider a partition of $S(\xi):=T^{-\top} N_{0}(\xi)$ given by $S(\xi)=\operatorname{col}\left(\mathcal{I}_{1}(\xi), \mathcal{I}_{2}(\xi)\right)$, where $\mathcal{I}_{1} \in \mathbb{R}^{l_{1} \times l}[\xi]$ and $\mathcal{I}_{2} \in \mathbb{R}^{\left(l-l_{1}\right) \times l}[\xi]$.

We have

$$
\left[\begin{array}{c}
R(\xi) \\
S(\xi)
\end{array}\right]=\left[\begin{array}{cc}
T & 0 \\
0 & T^{-\top}
\end{array}\right]\left[\begin{array}{c}
N_{1}(\xi) \\
N_{0}(\xi)
\end{array}\right]=\left[\begin{array}{c}
Y_{1}(\xi) \\
0_{\left(l-l_{1}\right) \times l} \\
\mathcal{I}_{1}(\xi) \\
\mathcal{I}_{2}(\xi)
\end{array}\right]
$$

Now observe that

$$
E_{1}(\zeta, \eta)=\frac{\zeta N_{1}(\zeta)^{\top} N_{0}(\eta)+N_{0}(\zeta)^{\top} N_{1}(\eta) \eta}{2(\zeta+\eta)}=\frac{\zeta Y_{1}(\zeta)^{\top} \mathcal{I}_{1}(\eta)+\mathcal{I}_{1}(\zeta)^{\top} Y_{1}(\eta) \eta}{2(\zeta+\eta)}
$$

We make use of Lemma 17 to prove that there exists a unimodular matrix $H \in$ $\mathbb{R}^{l \times l}[\xi]$ such that

$$
\begin{aligned}
& \mathcal{I}_{1} H=\left[\begin{array}{ll}
\tilde{N}_{0} & 0_{l_{1} \times\left(l-l_{1}\right)}
\end{array}\right], \\
& Y_{1} H=\left[\begin{array}{ll}
\tilde{N}_{1} & 0_{l_{1} \times\left(l-l_{1}\right)}
\end{array}\right]
\end{aligned}
$$

with $\tilde{N}_{0}, \tilde{N}_{1} \in \mathbb{R}^{l_{1} \times l_{1}}[\xi], \tilde{N}_{0}, \tilde{N}_{0}(0)$ nonsingular, and $\operatorname{col}\left(\tilde{N}_{1}, \tilde{N}_{0}\right)$ observable. This will enable us to prove that transformation (13) exists. 
Lemma 17. Let $l, l_{1} \in \mathbb{Z}_{+}$be such that $l_{1}<$ l. Consider matrices $R, S \in \mathbb{R}^{l \times l}[\xi]$, $Y_{1}, \mathcal{I}_{1} \in \mathbb{R}^{l_{1} \times l}[\xi], \mathcal{I}_{2} \in \mathbb{R}^{\left(l-l_{1}\right) \times l}[\xi]$ related by

$$
\left[\begin{array}{c}
R(\xi) \\
S(\xi)
\end{array}\right]=\left[\begin{array}{c}
Y_{1}(\xi) \\
0_{\left(l-l_{1}\right) \times l} \\
\mathcal{I}_{1}(\xi) \\
\mathcal{I}_{2}(\xi)
\end{array}\right] .
$$

Assume that $\operatorname{col}(R, S)$ is observable, $S$ and $S(0)$ are nonsingular, and $\xi R(\xi) S(\xi)^{-1}$ is $L P R$. Then there exists a unimodular matrix $H \in \mathbb{R}^{l \times l}[\xi]$ such that

$$
\begin{aligned}
& \mathcal{I}_{1} H=\left[\begin{array}{ll}
\tilde{N}_{0} & 0_{l_{1} \times\left(l-l_{1}\right)}
\end{array}\right], \\
& Y_{1} H=\left[\begin{array}{ll}
\tilde{N}_{1} & 0_{l_{1} \times\left(l-l_{1}\right)}
\end{array}\right]
\end{aligned}
$$

with $\tilde{N}_{0}, \tilde{N}_{1} \in \mathbb{R}^{l_{1} \times l_{1}}[\xi], \tilde{N}_{0}, \tilde{N}_{0}(0)$ nonsingular, and $\operatorname{col}\left(\tilde{N}_{1}, \tilde{N}_{0}\right)$ observable.

Proof. Since $S$ is nonsingular, it follows that $\mathcal{I}_{1}$ has full row rank $l_{1}$. This implies that there exists a unimodular $H \in \mathbb{R}^{l \times l}[\xi]$ such that (15) holds with $\tilde{N}_{0}$ nonsingular. Since $S(0)$ is nonsingular, it follows that $\mathcal{I}_{1}(0)$ has row rank equal to $l_{1}$, which in turn implies that $\tilde{N}_{0}(0)$ is nonsingular. We now prove that $Y_{1} H$ has its last $\left(l-l_{1}\right)$ columns filled with zeroes as in (16).

Since $\xi R(\xi) S(\xi)^{-1}$ is LPR, for all $\lambda \in \mathbb{C}$ with $\Re \mathrm{e}(\lambda)>0$,

$$
\bar{\lambda} S(\bar{\lambda})^{-\top} R(\bar{\lambda})^{\top}+\lambda R(\lambda) S(\lambda)^{-1} \geq 0 .
$$

Pre- and post-multiplying the above inequality by $S(\bar{\lambda})^{\top}$ and $S(\lambda)$, respectively, we obtain for all $\lambda \in \mathbb{C}$ with $\Re \mathrm{e}(\lambda)>0$,

$$
\bar{\lambda} R(\bar{\lambda})^{\top} S(\lambda)+\lambda S(\bar{\lambda})^{\top} R(\lambda) \geq 0 \quad \Longrightarrow \quad \bar{\lambda} Y_{1}(\bar{\lambda})^{\top} \mathcal{I}_{1}(\lambda)+\lambda \mathcal{I}_{1}(\bar{\lambda})^{\top} Y_{1}(\lambda) \geq 0
$$

Pre- and post-multiplying the above inequality by $H(\bar{\lambda})^{\top}$ and $H(\lambda)$, respectively, we obtain for all $\lambda \in \mathbb{C}$ with $\Re \mathrm{e}(\lambda)>0$,

$$
\bar{\lambda} H(\bar{\lambda})^{\top} Y_{1}(\bar{\lambda})^{\top} \mathcal{I}_{1}(\lambda) H(\lambda)+\lambda H(\bar{\lambda})^{\top} \mathcal{I}_{1}(\bar{\lambda})^{\top} Y_{1}(\lambda) H(\lambda) \geq 0 .
$$

Consider a partition of $Y_{1} H$ given by

$$
Y_{1} H=\left[\begin{array}{ll}
\tilde{N}_{1} & Y_{2}
\end{array}\right]
$$

with $\tilde{N}_{1} \in \mathbb{R}^{l_{1} \times l_{1}}[\xi]$ and $Y_{2} \in \mathbb{R}^{l_{1} \times\left(l-l_{1}\right)}[\xi]$. We now prove that $Y_{2}=0_{l_{1} \times\left(l-l_{1}\right)}$. From inequality (17), it follows that for all $\lambda \in \mathbb{C}$ with $\Re \mathrm{e}(\lambda)>0$,

$$
\Psi(\lambda):=\left[\begin{array}{cc}
\lambda \tilde{N}_{0}(\bar{\lambda})^{\top} \tilde{N}_{1}(\lambda)+\bar{\lambda}_{N_{1}(\bar{\lambda})^{\top} \tilde{N}_{0}(\lambda)} \lambda \tilde{N}_{0}(\bar{\lambda})^{\top} Y_{2}(\lambda) \\
\bar{\lambda} Y_{2}(\bar{\lambda})^{\top} \tilde{N}_{0}(\lambda) & 0_{\left(l-l_{1}\right) \times\left(l-l_{1}\right)}
\end{array}\right] \geq 0 .
$$

Note that since $\xi R(\xi) S(\xi)^{-1}$ is LPR and $\operatorname{col}(R, S)$ is observable, it follows that $S$ has no singularity in the open right half plane. Consequently, $\mathcal{I}_{1}(\lambda)$ and hence $\tilde{N}_{0}(\lambda)$ have full row rank for all $\lambda \in \mathbb{C}$ with $\Re \mathrm{e}(\lambda)>0$. The following reasoning is very similar to the argument used in the proof of [3, Theorem 5-11]. For $x \in \mathbb{C}^{l}$, define

$$
f(x):=x^{*} \Psi(\lambda) x
$$

Copyright $@$ by SIAM. Unauthorized reproduction of this article is prohibited. 
Partition $x$ as $x=\operatorname{col}\left(x_{1}, x_{2}\right)$ such that $x_{1} \in \mathbb{C}^{l_{1}}$ and $x_{2} \in \mathbb{C}^{l-l_{1}}$. Then

$$
\begin{aligned}
f(x)= & x_{1}^{*}\left(\lambda \tilde{N}_{0}(\bar{\lambda})^{\top} \tilde{N}_{1}(\lambda)+\bar{\lambda} \tilde{N}_{1}(\bar{\lambda})^{\top} \tilde{N}_{0}(\lambda)\right) x_{1}+x_{1}^{*}\left(\lambda \tilde{N}_{0}(\bar{\lambda})^{\top} Y_{2}(\lambda)\right) x_{2} \\
& +x_{2}^{*}\left(\bar{\lambda} Y_{2}(\bar{\lambda})^{\top} \tilde{N}_{0}(\lambda)\right) x_{1} \geq 0 .
\end{aligned}
$$

By varying $x_{2}$ and keeping $x_{1}$ fixed, we can make the sum of the second and third terms of the left-hand side of the above inequality as negative as desired, if it is nonzero. Thus, the sum of the second and third terms of the left-hand side of inequality (18) must be equal to zero for all $\lambda \in \mathbb{C}$ with $\Re \mathrm{e}(\lambda)>0$. By choosing $x_{1}$ and $x_{2}$ real and then choosing $x_{1}$ real and $x_{2}$ imaginary we obtain both $\lambda \tilde{N}_{0}(\bar{\lambda})^{\top} Y_{2}(\lambda)$ and $\bar{\lambda} Y_{2}(\bar{\lambda})^{\top} \tilde{N}_{0}(\lambda)$ to be equal to zero for all $\lambda \in \mathbb{C}$ with $\Re \mathrm{e}(\lambda)>0$. Since $\tilde{N}_{0}(\lambda)$ has full row rank for all $\lambda \in \mathbb{C}$ with $\Re \mathrm{e}(\lambda)>0$, it follows that $Y_{2}(\lambda)=0$ for all $\lambda \in \mathbb{C}$ with $\Re \mathrm{e}(\lambda)>0$. Consequently $Y_{2}(\xi)=0$ as a polynomial matrix.

We now prove that $\operatorname{col}\left(\tilde{N}_{1}, \tilde{N}_{0}\right)$ is observable. Assume by contradiction that there exists a $\lambda \in \mathbb{C}$ such that $\tilde{N}_{1}(\lambda) v_{1}=0$ and $\tilde{N}_{0}(\lambda) v_{1}=0$ for some $v_{1} \in \mathbb{C}^{l_{1}}$. Now define $G:=\mathcal{I}_{2} H$ and partition $G$ as $G=:\left[\begin{array}{ll}G_{1} & G_{2}\end{array}\right]$, where $G_{1} \in \mathbb{R}^{\left(l-l_{1}\right) \times l_{1}}[\xi]$ and $G_{2} \in$ $\mathbb{R}^{\left(l-l_{1}\right) \times\left(l-l_{1}\right)}[\xi]$. Note that since $\operatorname{col}(R, S)$ is observable, $\operatorname{col}(R(\lambda) H(\lambda), S(\lambda) H(\lambda))$ has full column rank. Observe that

$$
\left[\begin{array}{c}
R(\lambda) H(\lambda) \\
S(\lambda) H(\lambda)
\end{array}\right]=\left[\begin{array}{cc}
\tilde{N}_{0}(\lambda) & 0_{l_{1} \times\left(l-l_{1}\right)} \\
0_{\left(l-l_{1}\right) \times l_{1}} & 0_{\left(l-l_{1}\right) \times\left(l-l_{1}\right)} \\
\tilde{N}_{1}(\lambda) & 0_{l_{1} \times\left(l-l_{1}\right)} \\
G_{1}(\lambda) & G_{2}(\lambda)
\end{array}\right] .
$$

This implies that $G_{2}(\lambda)$ is nonsingular. Consequently, there exists a $v_{2} \in \mathbb{C}^{\left(l-l_{1}\right)}$ such that $G_{1}(\lambda) v_{1}=-G_{2}(\lambda) v_{2}$. Define $v_{3}:=\operatorname{col}\left(v_{1}, v_{2}\right)$ and $v:=H(\lambda) v_{3}$. It can be easily verified that $S(\lambda) v=0$ and $R(\lambda) v=0$. This is a contradiction since $\operatorname{col}(R, S)$ is observable. This proves that $\operatorname{col}\left(\tilde{N}_{1}, \tilde{N}_{0}\right)$ is observable.

Observe that since $\xi N_{1}(\xi) N_{0}(\xi)^{-1}$ is LPR,

$$
\xi R(\xi) S(\xi)^{-1}=\xi T N_{1}(\xi)\left(T^{-\top} N_{0}(\xi)\right)^{-1}=T\left(\xi N_{1}(\xi) N_{0}(\xi)^{-1}\right) T^{\top} \text { is also LPR. }
$$

Also note that since $\operatorname{col}\left(N_{1}, N_{0}\right)$ is observable, so is $\operatorname{col}(R, S)$. This implies the existence of a unimodular $H \in \mathbb{R}^{l \times l}[\xi]$ such that (15) and (16) hold with $\tilde{N}_{0}, \tilde{N}_{1} \in$ $\mathbb{R}^{l_{1} \times l_{1}}[\xi], \tilde{N}_{0}, \tilde{N}_{0}(0)$ nonsingular and $\operatorname{col}\left(\tilde{N}_{1}, \tilde{N}_{0}\right)$ observable.

It is a matter of straightforward verification to check that $R S^{-1}=\tilde{N}_{1}\left(\tilde{N}_{0}\right)^{-1}$. Consequently $\xi \tilde{N}_{1}(\xi)\left(\tilde{N}_{0}(\xi)\right)^{-1}$ is LPR and strictly proper. Since $Y_{1}$ has full row $\operatorname{rank} l_{1}, \operatorname{det}\left(\tilde{N}_{1}\right) \neq 0$.

Define

$$
\tilde{E}_{1}(\zeta, \eta):=\frac{\zeta \tilde{N}_{1}(\zeta)^{\top} \tilde{N}_{0}(\eta)+\tilde{N}_{0}(\zeta)^{\top} \tilde{N}_{1}(\eta) \eta}{2(\zeta+\eta)}
$$

From (14), it now follows that

$$
\begin{aligned}
E_{1}(\zeta, \eta) & =\frac{\zeta Y_{1}(\zeta)^{\top} \mathcal{I}_{1}(\eta)+\mathcal{I}_{1}(\zeta)^{\top} Y_{1}(\eta) \eta}{2(\zeta+\eta)} \\
& =H(\zeta)^{-\top}\left[\begin{array}{cc}
\tilde{E}_{1}(\zeta, \eta) & 0_{l_{1} \times\left(l-l_{1}\right)} \\
0_{\left(l-l_{1}\right) \times l_{1}} & 0_{\left(l-l_{1}\right) \times\left(l-l_{1}\right)}
\end{array}\right] H(\eta)^{-1} .
\end{aligned}
$$

Copyright (c) by SIAM. Unauthorized reproduction of this article is prohibited. 
Now as explained for Case 1 , we can obtain $N_{2} \in \mathbb{R}^{l_{1} \times l_{1}}[\xi]$ and positive definite $\tilde{A}_{1}, \tilde{B}_{1} \in \mathbb{R}_{s}^{l_{1} \times l_{1}}$ such that $\tilde{F}_{2}(\xi):=\xi N_{2}(\xi) \tilde{N}_{1}(\xi)^{-1}$ is LPR, $\tilde{Z}_{2}:=N_{2} \tilde{N}_{1}^{-1}$ is strictly proper and obeys $\tilde{Z}_{2}(\xi)=\tilde{Z}_{2}(-\xi)$, and moreover

$$
\tilde{N}_{0}(\xi)=\left(\tilde{A}_{1} \xi^{2}+\tilde{B}_{1}\right) \tilde{N}_{1}(\xi)-N_{2}(\xi)
$$

$\tilde{E}_{1}(\zeta, \eta)=\frac{1}{2}\left(\tilde{N}_{1}(\zeta)^{\top}\left(\tilde{A}_{1} \zeta \eta+\tilde{B}_{1}\right) \tilde{N}_{1}(\eta)-\tilde{N}_{1}(\zeta)^{\top} N_{2}(\eta)-N_{2}(\zeta)^{\top} \tilde{N}_{1}(\eta)\right)+E_{2}(\zeta, \eta)$,

where

$$
E_{2}(\zeta, \eta):=\frac{\zeta N_{2}(\zeta)^{\top} \tilde{N}_{1}(\eta)+\tilde{N}_{1}(\zeta)^{\top} N_{2}(\eta) \eta}{2(\zeta+\eta)}
$$

From the argument used in proving Case 1 it follows that $\tilde{N}_{1}(0)$ has full rank. Since $\operatorname{col}\left(\tilde{N}_{1}, \tilde{N}_{0}\right)$ is observable, from (19) it follows that $\operatorname{col}\left(N_{2}, \tilde{N}_{1}\right)$ is also observable.

After having examined Case 1 and Case 2 separately, we can now proceed with the rest of the proof.

If $N_{2} \neq 0$, based on whether $N_{2}$ is invertible or not, we repeat the steps mentioned under Case 1 or Case 2, respectively, with the new value of $N_{1}$ assigned as $N_{2}$ and of $N_{0}$ assigned as $N_{1}$ or $\tilde{N}_{1}$ depending on whether Case 1 or Case 2 was performed in the previous step. In this way, we expand the expression for $E^{\prime}$. This process continues until we obtain $N_{2}=0$, as we now prove.

LEMma 18. With the same notation as above, after a finite number of steps $N_{2}=0$.

Proof. If we go through the steps of Case 1, since $N_{2} N_{1}^{-1}$ and $N_{1} N_{0}^{-1}$ are strictly proper, every column of $N_{2}$ has degree less than that of the corresponding column of $N_{1}$ which is less than that of the corresponding column of $N_{0}$. Therefore

$$
\begin{aligned}
& \operatorname{deg}\left(\operatorname{det}\left(N_{0}\right)\right)-\operatorname{deg}\left(\operatorname{det}\left(N_{1}\right)\right) \geq 1, \\
& \operatorname{deg}\left(\operatorname{det}\left(N_{1}\right)\right)-\operatorname{deg}\left(\operatorname{det}\left(N_{2}\right)\right) \geq 1 .
\end{aligned}
$$

If we go through the steps of Case 2 , since $N_{2} \tilde{N}_{1}^{-1}$ and $\tilde{N}_{1} \tilde{N}_{0}^{-1}$ are strictly proper, it is a matter of straightforward verification to check that

$$
\operatorname{deg}\left(\operatorname{det}\left(N_{0}\right)\right) \geq \operatorname{deg}\left(\operatorname{det}\left(\tilde{N}_{0}\right)\right)>\operatorname{deg}\left(\operatorname{det}\left(\tilde{N}_{1}\right)\right)>\operatorname{deg}\left(\operatorname{det}\left(N_{2}\right)\right) .
$$

This implies that

$$
\begin{aligned}
& \operatorname{deg}\left(\operatorname{det}\left(N_{0}\right)\right)-\operatorname{deg}\left(\operatorname{det}\left(\tilde{N}_{1}\right)\right) \geq 1, \\
& \operatorname{deg}\left(\operatorname{det}\left(\tilde{N}_{1}\right)\right)-\operatorname{deg}\left(\operatorname{det}\left(N_{2}\right)\right) \geq 1 .
\end{aligned}
$$

Since Case 1 or Case 2 will be repeated as long as $N_{2} \neq 0$, at a particular step, we are bound to get $N_{2}=0$.

To conclude the proof of the theorem, we now organize and complete the argument given above in a detailed procedure to construct matrices $X, M$, and $K$ satisfying statements (i) and (ii).

Algorithm 19. Input: $N_{0}, D_{0} \in \mathbb{R}^{l \times l}[\xi]$ defining an observable image representation $w=\operatorname{col}\left(N_{0}\left(\frac{d}{d t}\right) \ell, D_{0}\left(\frac{d}{d t}\right) \ell\right)$ of a lossless negative imaginary behavior $\mathfrak{B}$ with Assumptions 1, 2, 3, and 4 in the statement of Theorem 15.

Output: A block tridiagonal real matrix $K$ with $K=K^{\top}>0$, a block-diagonal matrix $M$ with $M=M^{\top}>0$, and $X \in \mathbb{R}^{\bullet \times l}[\xi]$ satisfying (i) and (ii) of Theorem 15 . 
1: Compute $A_{0}, B_{0}$, and $N_{1}$ using the following formulae:

$$
\begin{gathered}
A_{0}=\lim _{\xi \rightarrow \infty} \frac{Z(\xi)^{-1}}{\xi^{2}} ; \quad B_{0}=\lim _{\xi \rightarrow \infty}\left(Z(\xi)^{-1}-A_{0} \xi^{2}\right) ; \\
N_{1}(\xi)=\left(A_{0} \xi^{2}+B_{0}\right) N_{0}(\xi)-D_{0}(\xi)
\end{gathered}
$$

2: Assign $P_{0}=N_{0}, Q_{0}=D_{0}, X_{0}=P_{0}, M_{0}=A_{0}, K_{0,0}=B_{0}$ and $i=1$.

3: While $N_{1} \neq 0$, do\{

4: $\quad$ Assign $l=$ number of rows of $N_{1}$.

5: $\quad$ If $\operatorname{det}\left(N_{1}\right) \neq 0,\{$

6: $\quad$ Compute $A_{1}, B_{1}$, and $N_{2}$ using the following formulae:

$$
\begin{gathered}
A_{1}=\lim _{\xi \rightarrow \infty} \frac{N_{0}(\xi) N_{1}(\xi)^{-1}}{\xi^{2}} ; \quad B_{1}=\lim _{\xi \rightarrow \infty}\left(N_{0}(\xi) N_{1}(\xi)^{-1}-A_{1} \xi^{2}\right) \\
N_{2}(\xi)=\left(A_{1} \xi^{2}+B_{1}\right) N_{1}(\xi)-N_{0}(\xi)
\end{gathered}
$$

7: $\quad$ Assign $P_{i}=N_{1}, Q_{i}=N_{0}, Y_{i}=P_{i}, M_{i}=A_{1}, K_{i, i}=B_{1}$,

$$
K_{i-1, i}=K_{i, i-1}=-I_{l} \text {. }
$$

8: $\quad$ Assign $X_{i}=$ matrix obtained by replacing $P_{i-1}$ in $X_{i-1}$ with $Y_{i}$, and assign $N_{0}=N_{1}$. $\}$

9: $\quad$ Else $\left\{\right.$ as explained under Case 2, compute $T, H, \tilde{N}_{0}$, and $\tilde{N}_{1}$.

10: $\quad$ Compute $\tilde{A}_{1}, \tilde{B}_{1}$, and $N_{2}$ using the following formulae:

$$
\begin{gathered}
\tilde{A}_{1}=\lim _{\xi \rightarrow \infty} \frac{\tilde{N}_{0}(\xi) \tilde{N}_{1}(\xi)^{-1}}{\xi^{2}} ; \quad \tilde{B}_{1}=\lim _{\xi \rightarrow \infty}\left(\tilde{N}_{0}(\xi) \tilde{N}_{1}(\xi)^{-1}-\tilde{A}_{1} \xi^{2}\right) \\
N_{2}(\xi)=\left(\tilde{A}_{1} \xi^{2}+\tilde{B}_{1}\right) \tilde{N}_{1}(\xi)-\tilde{N}_{0}(\xi)
\end{gathered}
$$

11: $\quad$ Assign $\left.P_{i}=\tilde{N}_{1}, Q_{i}=\tilde{N}_{0}, Y_{i}=\left[\begin{array}{cc}P_{i} & 0_{l_{1} \times\left(l-l_{1}\right)} \\ & 0_{\left(l-l_{1}\right) \times l}\end{array}\right] H^{-1}\right]$,

$$
\begin{aligned}
T_{1} & =T^{-1} \operatorname{col}\left(I_{l_{1}}, 0_{\left(l-l_{1}\right) \times l_{1}}\right), \quad M_{i}=\tilde{A}_{1}, \\
K_{i, i} & =\tilde{B}_{1}, \quad K_{i, i-1}=-T_{1}^{\top}, \quad K_{i-1, i}=-T_{1} .
\end{aligned}
$$

12: $\quad$ Assign $W_{i}=$ the matrix obtained by replacing $P_{i-1}$ in $X_{i-1}$ with $Y_{i}$.

13: $\quad$ Assign $\left.X_{i}=\left[\begin{array}{ll}I_{l_{1}} & 0_{l_{1} \times\left(l-l_{1}\right)}\end{array}\right] W_{i}, N_{0}=\tilde{N}_{1} \cdot\right\}$

14: $\quad$ Assign $N_{1}=N_{2}$ and $\left.i=i+1.\right\}$

15: Assign $n=i-1$.

16: Assign $X=\operatorname{col}\left(X_{0}, X_{1}, \ldots, X_{n}\right) ; M=\operatorname{diag}\left(M_{0}, M_{1}, \ldots, M_{n}\right)$.

17: Assign

$$
K=\left[\begin{array}{cccccc}
K_{0,0} & K_{0,1} & 0 & 0 & \cdots & 0 \\
K_{1,0} & K_{1,1} & K_{1,2} & 0 & \cdots & 0 \\
0 & K_{2,1} & K_{2,2} & K_{2,3} & \cdots & 0 \\
\vdots & \ddots & \ddots & \ddots & \ddots & \vdots \\
0 & \cdots & 0 & K_{n-1, n-2} & K_{n-1, n-1} & K_{n-1, n} \\
0 & \cdots & \cdots & 0 & K_{n, n-1} & K_{n, n}
\end{array}\right]
$$

Copyright (c) by SIAM. Unauthorized reproduction of this article is prohibited. 
It can be verified that $E^{\prime}(\zeta, \eta)=\frac{1}{2}\left(X(\zeta)^{T} K X(\eta)+\zeta \eta X(\zeta)^{T} M X(\eta)\right)$. The fact that $M=M^{\top}>0$ follows from $A_{i}$ and $\tilde{A}_{i}$ being positive definite; moreover, observe that $K=K^{\top}$. We make use of the following lemma to prove that $K>0$.

Lemma 20. Let $K$ have a block-tridiagonal structure as in (20). Assume that $K$ is symmetric and its diagonal elements are positive definite. For $i=0, \ldots, n$, define

$$
\Gamma_{i}= \begin{cases}K_{n, n} & \text { if } i=n, \\ K_{i, i}-K_{i, i+1} \Gamma_{i+1}^{-1} K_{i, i+1}^{\top} & \text { if } i<n .\end{cases}
$$

If $\Gamma_{i}>0$ for $i=0, \ldots, n$, then $K$ is positive definite.

Proof. For $i=0, \ldots, n$, factor $\Gamma_{i}=\Lambda_{i}^{\top} \Lambda_{i}$ with $\Lambda_{i}$ square and nonsingular, $i=0, \ldots, n$. Define $Q_{i}:=\Lambda_{i}^{-\top} K_{i, i-1}$ for $i=1, \ldots, n$ and

$$
L:=\left[\begin{array}{cccccc}
\Lambda_{0} & 0 & 0 & 0 & \cdots & 0 \\
Q_{1} & \Lambda_{1} & 0 & 0 & \cdots & 0 \\
0 & Q_{2} & \Lambda_{2} & 0 & \cdots & 0 \\
\vdots & \ddots & \ddots & \ddots & \ddots & \vdots \\
0 & \cdots & 0 & Q_{n-1} & \Lambda_{n-1} & 0 \\
0 & \cdots & \cdots & 0 & Q_{n} & \Lambda_{n}
\end{array}\right] .
$$

It is easy to verify that $K=L^{\top} L$. Since $\operatorname{det}\left(\Lambda_{i}\right) \neq 0$ for $i=0, \ldots, n$, it follows that $\operatorname{det}(L) \neq 0$. This implies that $K$ is positive definite.

We now use this lemma to prove that the matrix $K$ produced by the algorithm is positive definite. Define $Z_{i}:=Q_{i} P_{i}^{-1}$ and $F_{i}(\xi):=\frac{Z_{i}(\xi)}{\xi}$ for $i=0, \ldots, n$. Since $F_{i}$ is LPR and $Q_{i}(0)$ has full rank, from Theorem 8 it follows that $Z_{i}(0)$ is positive definite for $i=0, \ldots, n$. It can be verified that

$$
Z_{i}(\xi)=M_{i} \xi^{2}+K_{i, i}-K_{i, i+1} Z_{i+1}^{-1}(\xi) K_{i, i+1}^{\top}
$$

This implies that

$$
Z_{i}(0)=K_{i, i}-K_{i, i+1} Z_{i+1}^{-1}(0) K_{i, i+1}^{\top} ;
$$

moreover since $P_{n+1}(\xi)$ and hence $Z_{n+1}^{-1}(\xi)$ are equal to zero, we obtain $Z_{n}(0)=K_{n, n}$. Now apply Lemma 20 with $\Gamma_{i}=Z_{i}(0)$; it follows that $K>0$.

Equation (10) can now be verified by following the steps of the algorithm given just before Lemma 20. Since this equation is of first order in $q$ and $p$ and of zeroth order in $w$, it is easy to see that it is a state space representation of $\mathfrak{B}$. It follows that $\operatorname{col}(X(\xi), \xi M X(\xi))$ is a state map for $\mathfrak{B}$.

COROllary 21. Consider a lossless negative imaginary behavior $\mathfrak{B}$ with an observable image representation $w=\left(\begin{array}{c}N_{0}\left(\frac{d}{d t}\right) \\ D_{0}\left(\frac{d}{d t}\right)\end{array}\right) \ell$ with $N_{0}, D_{0} \in \mathbb{R}^{l \times l}[\xi]$ and define $y:=$ $N_{0}\left(\frac{d}{d t}\right) \ell, u:=D_{0}\left(\frac{d}{d t}\right) \ell$. Under the same assumptions of Theorem 15, there exists $N \in \mathbb{Z}_{+}$such that $\mathfrak{B}$ has a state space representation of the form

$$
\begin{aligned}
\frac{d}{d t} x & =\Omega^{-1} Q x+B_{1} u, \\
y & =B_{1}^{\top} \Omega x
\end{aligned}
$$

Copyright $@$ by SIAM. Unauthorized reproduction of this article is prohibited. 
where $\Omega \in \mathbb{R}^{2 N \times 2 N}$ is skew symmetric, $B_{1} \in \mathbb{R}^{2 N \times l}$, and $Q \in \mathbb{R}^{2 N \times 2 N}$ is positive definite such that $x^{\top} Q x$ is the principal energy associated with $w \in \mathfrak{B}$.

Proof. With reference to Theorem 15, define the following:

$$
\Omega:=\left[\begin{array}{cc}
0_{N} & -I_{N} \\
I_{N} & 0_{N}
\end{array}\right] ; \quad Q:=\operatorname{diag}\left(K, M^{-1}\right) ; \quad x:=\operatorname{col}(q, p) ; \quad B_{1}:=\operatorname{col}\left(0_{N \times l}, B\right) .
$$

Then the state space representation (10) reduces to (21) and it can be verified that the principal energy associated with $w$ is indeed $x^{\top} Q x$.

Remark 22. Representation (21) is the same as the representation of timereversible Hamiltonian systems obtained in [7]. Indeed the transfer function $G$ of a lossless negative imaginary behavior obeys $G(s)=G(-s)=G(-s)^{\top}$. Incidentally the results of this section show that since we also have $K=K^{\top}>0$ and $M=M^{\top}>0$ in (10), a lossless negative imaginary behavior $\mathfrak{B}=\operatorname{Im}\left(\begin{array}{l}N_{0}\left(\frac{d}{d t}\right) \\ D_{0}\left(\frac{d}{d t}\right)\end{array}\right)$ with the properties mentioned in Theorem 15 belongs to the class of Hamiltonian systems for which a model reduction procedure has been described in [8].

Given below is an example where the method sketched in the proof of Theorem 15 is used to obtain a minimal state map for a given lossless negative imaginary behavior.

Example 23. Consider

$$
D_{0}(\xi):=\left[\begin{array}{cc}
5 \xi^{4}+22 \xi^{2}+5 & \xi^{4}+2 \xi^{2}-7 \\
\xi^{4}+2 \xi^{2}-7 & 2 \xi^{4}+11 \xi^{2}+10
\end{array}\right] \text { and } N_{0}(\xi):=\left[\begin{array}{cc}
\xi^{2}+4 & 0 \\
0 & \xi^{2}+4
\end{array}\right] .
$$

It can be verified that $N_{0}$ and $D_{0}$ are both invertible, $D_{0}(0)$ has full rank, $Z:=N_{0} D_{0}^{-1}$ is strictly proper, $Z(\xi)=Z(-\xi)$, and the elements of $Z$ have poles at $\pm 2.1754 \mathrm{~J}$, $\pm 2.0209 \mathrm{~J}, \pm 1.3176 \mathrm{~J}, \pm 0.0575 \mathrm{~J}$, which implies that all elements of $Z$ are analytic in the open right half plane. It can also be verified that $L:=\operatorname{col}\left(N_{0}, D_{0}\right)$ induces an observable image representation; consequently there exists $F \in \mathbb{R}^{2 \times 4}[\xi]$ such that $F(\xi) L(\xi)=I_{2}$. This implies that for every trajectory

$$
w=L\left(\frac{d}{d t}\right) \ell
$$

that belongs to the behavior $\mathfrak{B}:=\operatorname{Im}\left(L\left(\frac{d}{d t}\right)\right)$, we have $\ell=F\left(\frac{d}{d t}\right) w$. Note that in (22), the dimension of the latent variable $\ell$ is $l=2$. It can be verified that

$$
E^{\prime}(\zeta, \eta):=\frac{\zeta N_{0}(\zeta)^{\top} D_{0}(\eta)+D_{0}(\zeta)^{\top} N_{0}(\eta) \eta}{2(\zeta+\eta)}
$$

induces a positive QDF. Define $E(\zeta, \eta):=F(\zeta)^{\top} E^{\prime}(\zeta, \eta) F(\eta)$, and observe that $E^{\prime}(\zeta, \eta)=L(\zeta)^{\top} E(\zeta, \eta) L(\eta)$. This implies that for $w$ given by $(22), Q_{E}(w)=Q_{E^{\prime}}(\ell)$. This in turn implies that $Q_{E}(w) \stackrel{\mathfrak{B}}{>} 0$. We have

$$
(\zeta+\eta) E^{\prime}(\zeta, \eta)=L(\zeta)^{\top} J(\zeta, \eta) L(\eta)=(\zeta+\eta) L(\zeta)^{\top} E(\zeta, \eta) L(\eta)
$$

This implies that for every trajectory $w \in \mathfrak{B}, Q_{J}(w)=\frac{d}{d t} Q_{E}(w)$. It follows that $\mathfrak{B}$ is lossless negative imaginary and all other conditions of Theorem 15 are obeyed. Therefore, we can follow the steps given in the proof of the theorem in order to obtain matrices $M$ and $K$ and a state map $X^{\prime}(\xi)$ that gives rise to a state space 
representation (10) for $\mathfrak{B}$. This gives

$$
\begin{gathered}
M=\left[\begin{array}{cccc}
5 & 1 & 0 & 0 \\
1 & 2 & 0 & 0 \\
0 & 0 & 0.4 & 0.2 \\
0 & 0 & 0.2 & 0.6
\end{array}\right] ; \quad K=\left[\begin{array}{cccc}
2 & -2 & -1 & 0 \\
-2 & 3 & 0 & -1 \\
-1 & 0 & 1.6 & 0.8 \\
0 & -1 & 0.8 & 2.4
\end{array}\right] ; \\
X^{\prime}(\xi)=\left[\begin{array}{cc}
\xi^{2}+4 & 0 \\
0 & \xi^{2}+4 \\
3 & -1 \\
-1 & 2 \\
5 \xi\left(\xi^{2}+4\right) & \xi\left(\xi^{2}+4\right) \\
\xi\left(\xi^{2}+4\right) & 2 \xi\left(\xi^{2}+4\right) \\
\xi & 0 \\
0 & \xi
\end{array}\right] .
\end{gathered}
$$

5. SISO lossless negative imaginary behaviors and synthesis of SISO lossless mechanical systems. Let

$$
\mathfrak{B}=\operatorname{Im}\left(\begin{array}{c}
n\left(\frac{d}{d t}\right) \\
d\left(\frac{d}{d t}\right)
\end{array}\right)
$$

be an observable image representation of an SISO lossless negative imaginary behavior with $\operatorname{deg}(d)>\operatorname{deg}(n)$ and external variables $y$ (output) and $u$ (input). In this section, we show that in the SISO case considerable simplifications occur to the result of Theorem 15 and that Algorithm 19 can be used to produce in a straightforward way a mechanical synthesis of a system with transfer function $\frac{n}{d}$.

We first show that assumption (1) of Theorem 15, i.e., that $d$ has no root at 0 , can be deduced from the fact that the transfer function is SISO and LPR. Assume by contradiction that $d$ has a root at 0 . Since $\mathfrak{B}$ is lossless negative imaginary, it follows that $d n^{-1}$ is of the form given in (6) with $B>0$. This implies that $n$ also has a root at 0 , which in turn implies that representation (23) is not observable. This proves that $d(0) \neq 0$, as required.

Note also that from the expansion (6) and the observability of $\operatorname{col}(n, d)$, it follows that both $n$ and $d$ are even and consequently that the McMillan degree $\mathrm{n}(\mathfrak{B})=\operatorname{deg}(d)$ is even.

Now consider that since the system is SISO, in the procedure stated in the proof of Theorem 15, only Case 1 can happen, and consequently considerable simplifications occur. In step 6 of Algorithm 19, note that $N_{0}$ and $N_{1}$ are polynomials and hence $A_{1}$ is the leading coefficient of the quotient $q$ of the Euclidean division of $N_{0}$ by $N_{1}$. From (6) and the expression for $B_{1}$ in step 6 of Algorithm 19, it follows that $B_{1}$ is equal to the constant term of $q$. It is also easy to see that $N_{2}$ is equal to the negative of the remainder $r$ of the Euclidean division of $N_{0}$ by $N_{1}$. Now from steps 7, 16, and 17 of Algorithm 19, it follows that the matrix $M$ is diagonal and $K$ is tridiagonal with positive constants along the main diagonal and each element on the two neighboring subdiagonals being equal to -1 .

We now prove that the state map for $\mathfrak{B}$ that we obtain using Algorithm 19 is minimal. Note that every time we go through steps 6,7 , and 8 , the degree of $N_{0}$ reduces by 2 . This implies that the dimension of $X$ obtained in step 16 is $N=\mathrm{n}(\mathfrak{B}) / 2$ and the dimension of the state map obtained by the algorithm is $\mathrm{n}(\mathfrak{B})$, which in turn implies that the algorithm leads to a minimal state map for $\mathfrak{B}$. 


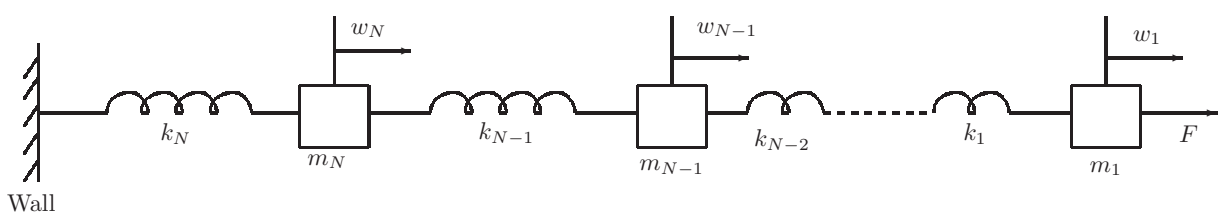

FIG. 1. A spring-mass system.

Having concluded the discussion of the simplifications occurring in the SISO case, we now show how to compute a mechanical realization of $\mathfrak{B}$, i.e., a mechanical system consisting of masses and springs with an external force acting on one of the masses such that there exists a mass whose displacement from its equilibrium position, together with the external force, defines a set of trajectories equal to $\mathfrak{B}$.

We use the procedure of Algorithm 19, obtaining a diagonal matrix $M$, a tridiagonal matrix $K$, and a polynomial vector $X$ with dimension $N$. Let $b_{i}$ denote the ith diagonal entry of $K$. Define $x_{1}:=X\left(\frac{d}{d t}\right) \ell$ and $x_{2}:=\frac{d}{d t}\left(M X\left(\frac{d}{d t}\right)\right) \ell$. Let $B$ denote the column vector of dimension $N$ whose first element is 1 and the rest are equal to zero. Then we have the following state space representation for $\mathfrak{B}$ :

$$
\begin{aligned}
\frac{d}{d t}\left[\begin{array}{l}
x_{1} \\
x_{2}
\end{array}\right] & =\left[\begin{array}{cc}
0_{N} & M^{-1} \\
-K & 0_{N}
\end{array}\right]\left[\begin{array}{l}
x_{1} \\
x_{2}
\end{array}\right]+\left[\begin{array}{c}
0_{N \times 1} \\
B
\end{array}\right] u, \\
y & =B^{\top} x_{1} .
\end{aligned}
$$

We now derive from this representation one which leads directly to a mechanical realization of $\mathfrak{B}$. For $i=1, \ldots, N-1$, define $\delta_{i}:=$ leading principal ith minor of $K$ and $\delta_{0}:=1$, and define the diagonal matrix $D:=\operatorname{diag}\left(\delta_{0}, \delta_{1}, \ldots, \delta_{N-1}\right)$. Observe that since $K$ is positive definite, so is $D$. Define

$$
\mathcal{K}:=D K D ; \quad \mathcal{M}:=D M D ; \quad\left[\begin{array}{l}
q \\
p
\end{array}\right]:=\left[\begin{array}{cc}
D^{-1} & 0 \\
0 & D
\end{array}\right]\left[\begin{array}{l}
x_{1} \\
x_{2}
\end{array}\right] .
$$

We obtain the following state space representation in terms of the new state vector $\operatorname{col}(q, p)$ :

$$
\begin{aligned}
\frac{d}{d t}\left[\begin{array}{l}
q \\
p
\end{array}\right] & =\left[\begin{array}{cc}
0_{N} & \mathcal{M}^{-1} \\
-\mathcal{K} & 0_{N}
\end{array}\right]\left[\begin{array}{l}
q \\
p
\end{array}\right]+\left[\begin{array}{c}
0_{N \times 1} \\
B
\end{array}\right] u, \\
y & =B^{\top} q .
\end{aligned}
$$

Now define $\delta_{-1}:=0$ and $\delta_{N}:=0$. Observe that $\mathcal{M}$ is diagonal and $\mathcal{K}$ is tridiagonal with $\mathcal{K}_{i, i}=\delta_{i-1}^{2} b_{i}, \mathcal{K}_{i, i+1}=-\delta_{i-1} \delta_{i}$, and $\mathcal{K}_{i, i-1}=-\delta_{i-2} \delta_{i-1}$ for $i=1, \ldots, N$. It can be verified that

$$
\mathcal{K}_{i, i}=-\left(\mathcal{K}_{i, i+1}+\mathcal{K}_{i, i-1}\right)>0 \quad \text { for } \quad i=1, \ldots, N-1 .
$$

Now consider a mechanical spring-mass system consisting of $N$ springs with spring constants $k_{1}, k_{2}, \ldots, k_{N}$ and $N$ masses $m_{1}, m_{2}, \ldots, m_{N}$ interconnected to each other and to the wall as shown in Figure 1.

Assume that the springs and masses are constrained to move in a horizontal plane in a particular direction. In this direction, let $w_{i}$ denote the horizontal displacement of the ith mass with respect to the wall. Assume that a force $F$ acts on the first mass as shown in Figure 1.

Define $m_{i}:=$ ith diagonal entry of $\mathcal{M}$ for $1=1, \ldots, N ; k_{i}:=-\mathcal{K}_{i, i+1}$ for $i=$ $1,2, \ldots, N-1 ; k_{N}:=\mathcal{K}_{N, N}+\mathcal{K}_{N, N-1} ; F:=u$; and $w_{1}:=y$. Then, it can be verified that the equations of motion of the mechanical spring-mass system are given by (25) 
with $q=\operatorname{col}\left(w_{1}, w_{2}, \ldots, w_{N}\right)$ and (26) will be obeyed for the system. This implies that the system described in Figure 1 with parameters $m_{1}, m_{2}, \ldots, m_{N}$ and $k_{1}, k_{2}, \ldots, k_{N}$ is a mechanical realization of the given behavior $\mathfrak{B}$.

The simplification of Algorithm 19 for the case of SISO behaviors and its application for the synthesis of mechanical systems with lossless negative imaginary behaviors suggests to use Algorithm 24 also to compute a mechanical realization corresponding to an SISO lossless negative imaginary behavior.

AlgORITHM 24. Input: An SISO lossless negative imaginary behavior with an observable image representation $\mathfrak{B}=\operatorname{Im}\left(\operatorname{col}\left(n\left(\frac{d}{d t}\right), d\left(\frac{d}{d t}\right)\right)\right.$ with $\mathrm{n}(\mathfrak{B})=2 N=$ $\operatorname{deg}(d)>\operatorname{deg}(n)$.

Output: $A$ tridiagonal $\mathcal{K}$ and a diagonal $\mathcal{M}$ corresponding to a state representation of the form $(25)$ of $\mathfrak{B}$, a corresponding state map $X(\xi)$, and $\left\{m_{i}, k_{i}\right\}_{i=1, \ldots, N}$ corresponding to a mechanical realization of $\mathfrak{B}$ of the form shown in Figure 1.

1: For $(i=1$ to $N)$ do \{

2: $\quad$ Find the quotient $q$ and the remainder $r$ of the Euclidean division of $d$ by $n$.

3: $\quad$ Assign $a_{i}=$ the leading coefficient of $q$.

4: $\quad$ Assign $b_{i}=$ the constant term of $q$.

5: $\quad$ Assign $n_{i}:=n, d=n$, and $n=-r$.

6: Assign $M:=\operatorname{diag}\left(a_{1}, a_{2}, \ldots, a_{N}\right)$.

7: Assign $K:=\left[\begin{array}{cccccc}b_{1} & -1 & 0 & 0 & \cdots & 0 \\ -1 & b_{2} & -1 & 0 & \cdots & 0 \\ 0 & -1 & b_{3} & -1 & \cdots & 0 \\ \vdots & \ddots & \ddots & \ddots & \ddots & \vdots \\ 0 & \cdots & 0 & -1 & b_{N-1} & -1 \\ 0 & \cdots & \cdots & 0 & -1 & b_{N}\end{array}\right]$.

8: Assign $\delta_{0}:=1$ and for $(i=1$ to $N-1)$ assign $\delta_{i}:=$ determinant of the top left-most $(i \times i)$ block of $K_{1}$.

9: Assign $D:=\operatorname{diag}\left(\delta_{0}, \delta_{1}, \ldots, \delta_{N-1}\right)$.

10: Assign $\mathcal{K}:=D K D, \mathcal{M}:=D M D$.

11: Assign $x_{1}(\xi):=\operatorname{col}\left(n_{1}(\xi), n_{2}(\xi), \ldots, n_{N}(\xi)\right)$.

12: Compute $q(\xi)=D^{-1} x_{1}(\xi), X(\xi)=\operatorname{col}(q(\xi), \xi \mathcal{M} q(\xi))$.

13: For $(i=1$ to $N)$ assign $m_{i}=\mathcal{M}_{i, i}$.

14: Assign $k_{N}:=\mathcal{K}_{N, N}+\mathcal{K}_{N, N-1}$ and for $(i=1$ to $N-1)$, assign $k_{i}:=-\mathcal{K}_{i, i+1}$.

15: Output $\mathcal{M}, \mathcal{K}, X(\xi),\left\{m_{i}, k_{i}\right\}_{i=1, \ldots, N}$.

We now illustrate Algorithm 24 with an example.

Example 25. Let $\mathfrak{B}=\operatorname{Im}\left(\operatorname{col}\left(n\left(\frac{d}{d t}\right), d\left(\frac{d}{d t}\right)\right)\right)$ with $n(\xi)=\xi^{4}+4 \xi^{2}+3$ and $d(\xi)=2 \xi^{6}+13 \xi^{4}+22 \xi^{2}+8$. It can be verified that $\mathfrak{B}$ is lossless negative imaginary. Application of Algorithm 24 gives the following output:

$$
\begin{gathered}
\mathcal{K}=\left[\begin{array}{ccc}
5 & -5 & 0 \\
-5 & 14.0625 & -9.0625 \\
0 & -9.0625 & 24.5292
\end{array}\right] ; \quad \mathcal{M}=\left[\begin{array}{ccc}
2 & 0 & 0 \\
0 & 6.25 & 0 \\
0 & 0 & 14.0167
\end{array}\right] \\
X(\xi)=\left[\begin{array}{c}
\xi^{4}+4 \xi^{2}+3 \\
0.8 \xi^{2}+1.4 \\
0.5172 \\
2 \xi^{5}+8 \xi^{3}+6 \xi \\
5 \xi^{3}+8.75 \xi \\
7.2494 \xi
\end{array}\right]
\end{gathered}
$$

Copyright (c) by SIAM. Unauthorized reproduction of this article is prohibited. 
and the coefficients $k_{1}=5, k_{2}=9.0625, k_{3}=15.4667, m_{1}=2, m_{2}=6.25, m_{3}=$ 14.0167 .

6. Conclusions. We have presented an algorithm for the realization of a lossless negative behavior based on successive divisions of univariate square polynomial matrices. We have also sketched a method of synthesis of lossless mechanical systems based on our realization procedure. The algebra of univariate and bivariate polynomial matrices has been used throughout as a tool for proving many of the results of this paper.

We believe that the realization procedure presented in our paper is not relevant for the synthesis of LC circuits. The reason for this is that although a capacitor is an electrical analogue of a spring, an inductor is not an electrical analogue of a mass. The inductor is an electrical analogue of another mechanical component known as an inerter and described in the work of Malcolm Smith; see, e.g., [6]. Thus the standard mechanical-electrical analogies cannot be used in order to synthesize LC circuits using our realization procedure. Moreover the tridiagonal structure of $K$ and diagonal structure of $M$ are only suitable for the synthesis of mechanical networks of the type discussed in section 5 .

\section{REFERENCES}

[1] B. D. O. Anderson And S. Vongpanitlerd, Network Analysis and Synthesis: A Modern Systems Theory Approach, Prentice-Hall, Englewood Cliffs, NJ, 1973.

[2] T. Kailath, Linear Systems, Prentice-Hall, Englewood Cliffs, NJ, 1980.

[3] R. Newcomb, Linear Multiport Synthesis, McGraw-Hill, New York, 1966.

[4] J. W. Polderman and J. C. Willems, Introduction to Mathematical System Theory: A Behavioral Approach, Springer-Verlag, Berlin, 1997.

[5] P. Rapisarda and J. C. Willems, State maps for linear systems, SIAM J. Control Optim., 35 (1997), pp. 1053-1091.

[6] M. C. Smith, Synthesis of mechanical networks: The inerter, IEEE Trans. Automat. Control, 47 (2002), pp. 1648-1662.

[7] A. J. van Der Schaft, Time-reversible Hamiltonian systems, Systems Control Lett., 1 (1982), pp. 295-300.

[8] A. J. Van der Schaft and J. E. Oeloff, Model reduction of linear conservative mechanical systems, IEEE Trans. Automat. Control, 35 (1990), pp. 729-733.

[9] J. C. Willems and H. L. Trentelman, On quadratic differential forms, SIAM J. Control Optim., 36 (1998), pp. 1703-1749.

[10] J. Xiong, I. R. Peterson, and A. Lanzon, On lossless negative imaginary systems, in Proceedings of the 7th Asian Control Conference, Hong Kong, 2009, pp. 824-829.

Copyright (c) by SIAM. Unauthorized reproduction of this article is prohibited. 